\title{
Alcohol Cues Elicit Different Abnormalities in Brain Networks of Abstinent Men and Women with Alcohol Use Disorders
}

\author{
Kayle S. Sawyer, Ph.D.1,2,3,4, Marlene Oscar-Berman, Ph.D.1,2,3, Susan Mosher Ruiz, Ph.D. ${ }^{2}$, \\ Ksenija Marinkovic, Ph.D.5, Mary M. Valmas, Ph.D.2, and Gordon J. Harris, Ph.D.3,6 \\ ${ }^{1}$ Psychology Research Service, VA Boston Healthcare System, Boston, MA; \\ 2Department of Anatomy \& Neurobiology, Boston University School of Medicine, Boston, MA; \\ ${ }^{3}$ Department of Radiology, Massachusetts General Hospital, Boston, MA; \\ ${ }^{4}$ Sawyer Scientific, LLC, Boston, MA \\ ${ }^{5}$ Department of Psychology, San Diego State University, San Diego, CA; \\ ${ }^{6}$ Department of Radiology, Harvard Medical School, Boston, MA;
}

Corresponding Author: Kayle Sawyer, Ph.D.

Boston University School of Medicine, L-1004

72 East Concord Street

Boston, MA 02118

USA

Fax: +1 857-364-2214; Phone: +1 857-364-4005

Email: kslays@bu.edu

Keywords: Alcoholism; reward; fMRI; gender; memory; emotion

Running Title: Gender, brain responses, and alcohol

Highlights: Brain reward regions activate highly when individuals with a history of alcohol use disorders view alcoholic beverages.

The brain regions identified subserve vision, memory, and judgement.

Opposite abnormalities in activation patterns appeared for alcoholic men and women. 


\section{Abstract}

34 We employed fMRI in 84 men and women with and without a history of alcohol use disorders

35 (ALC and NC, respectively), to explore how gender interacts with alcoholism as reflected in

36 brain activity elicited by alcohol cues. Brain activation was measured in a working memory task

37 (delayed matching-to-sample) with emotional faces as the sample and match cues. During the

38 delay period, intervening distractors were either reward-salient cues (alcoholic beverages) or

39 neutral cues (nonalcoholic beverages or scrambled pictures). ALC women (ALCw) had higher

40 accuracy than ALC men (ALCm). Analyses of scans during the viewing of distractor images

41 revealed significant group-by-gender interactions. Compared to NC men, ALCm evidenced

42 lower activation contrast between reward-salient cues and neutral cues in default mode network

43 regions (including superior prefrontal and precuneus areas), while ALCw had more activation

44 than NC women. Similar interactions were observed for task-regions (including superior parietal,

45 lateral occipital, and prefrontal areas). Region of interest analyses showed that the ALC group

46 had significantly higher levels of activation throughout reward-related circuitry during alcohol

47 distractor interference than during scrambled picture interference. These results suggest that

48 abstinent ALCm and ALCw differ in processing reward-salient cues, which can impact treatment

49 and recovery. 


\section{Introduction}

Alcohol use disorders (AUD) have been associated with deficits in cognitive and emotional functions (Oscar-Berman et al., 2014). Because of their reward salience, alcohol cues

54 such as pictures of alcoholic beverages elicit attentional bias and brain activation in individuals

55 with AUD (Carter \& Tiffany, 1999; Goldstein \& Volkow, 2002; Schacht et al., 2013). Alcohol

cues induce a hyperattentive state with attention drawn to the rewarding stimuli (Townshend \&

57 Duka, 2001; Franken, 2003; Field \& Cox, 2008; Vollstädt-Klein et al., 2011). Therefore, the

cues selectively interfere with other cognitive abilities such as memory. Importantly, attentional

bias toward alcohol-related stimuli also has been associated with level of craving, consumption,

60 dependence, and physiological arousal (Sharma et al., 2001; Ryan, 2002; Field et al., 2004; Field

61 \& Eastwood, 2005; Bordnick et al., 2008; Sinha et al., 2009; Wiers et al., 2014; Sawyer et al.,

62 2015).

Functional magnetic resonance imaging (fMRI) studies of attentional biasing, and

64 specifically cue sensitivity, have often included either only men with and without a history of

65 AUD (ALC and NC groups), or groups of men and women with sample sizes too small to

66 examine gender effects (Fryer et al., 2013; Schacht et al., 2013). However, gender impacts the

67 ways in which alcohol affects the brain and behavior (Becker et al., 2017; Sawyer et al., 2017,

68 2018, 2019; Seitz et al., 2017; Rivas-Grajales et al., 2018; Hoffman et al., 2019; Kaag et al.,

69 2019; Fama et al., 2020; Verplaetse et al., 2021), due to interactions with physiological and

70 social factors (Ruiz \& Oscar-Berman, 2015; Mosher Ruiz et al., 2017). In the present study, we

71 examined gender differences using a delayed matching-to-sample (DMTS) task (Dolcos \&

72 McCarthy, 2006) with alcohol cues serving as distractor stimuli, in a cohort of ALC and NC men

73 and women (AUDm, AUDw, $\mathrm{NCm}$, and $\mathrm{NCw}$ ). The DMTS task requires an attention-demanding 
74 kind of memory called "working memory." In this study, the participants were required to

75 remember photographs of emotional faces while distracting pictures of alcoholic beverages,

76 nonalcoholic beverages, or scrambled images intervened during the delay period. We chose faces

77 as the sample stimuli for two primary reasons. First, in a previous study (Marinkovic et al., 2009)

78 we found that men with AUD had abnormally low brain activity in temporal limbic regions when

79 viewing faces, and second, we used the same dataset that we had acquired for a prior report

80 (Oscar-Berman et al., 2019) in which we described the brain's responses to the initial to-be-

81 encoded emotional faces phase (the sample) of the DMTS task. For the present study, the data

82 derived from the delay and match portions of the task allowed us to test the attentional biasing

83 effect, wherein we expected alcohol cues, more than other cue types, to impair performance on

84 memory for face identity.

Functional MRI tasks activate multiple brain networks, and abnormalities in the default mode network (DMN) have been implicated in AUD and in psychiatric disorders (Menon, 2011;

87 Zhang \& Volkow, 2019). The DMN has been observed to be more active during story telling,

88 reading and memory tasks, imagining future scenarios, self-reference, rumination, and when the

89 mind wanders while staring at a fixation cross during fMRI scanning (Tops et al., 2014; Beaty et

90 al., 2016; Buckner \& DiNicola, 2019). We refer to the DMN regions as fixation-regions because

91 they are more active during the idle delay intervals when the fixation stimulus is presented

92 between DMTS trials than during stimulus presentations. Vertex-wise analyses have revealed

93 that cortical fixation-regions include: (1) an "anterior hub," consisting of portions of the rostral

94 anterior cingulate cortex (ACC), ventromedial prefrontal cortex, and medial superior frontal

95 cortex; (2) a "posterior hub," which includes portions of the posterior cingulate and precuneus,

96 (3) the temporoparietal junction, which covers parts of the angular gyrus and inferior parietal 
97 lobule; and (4) the superior and middle temporal gyrus region (Margulies et al., 2016; Buckner

98 \& DiNicola, 2019; Uddin et al., 2019).

In addition to the DMN regions, we used vertex-wise analyses to examine task-regions,

100 which are more engaged during the DMTS task than while looking at unengaging fixation

101 crosses. Literature on distractor interference during working memory has suggested that task-

102 regions involve a distributed network including prefrontal cortex, along with dorsal and ventral

103 visual association cortex, which are necessary for attentional functioning (Loeber et al., 2009)

104 and for inhibiting distracting visual stimuli (Jha et al., 2004; Clapp et al., 2010). Additional task-

105 regions involved in attention, working memory, and emotional processing, include the dorsal

106 ACC and lateral prefrontal areas. The dorsal ACC in particular has been implicated in craving

107 and attentional biasing (Goldstein \& Volkow, 2011).

109 defined regions of interest (ROI) involved in alcohol cue exposure, distractor interference,

110 craving, reward processing and salience, or working memory for emotional faces. The first nine

111 ROI are the dorsolateral prefrontal cortex (DLPFC), ventrolateral prefrontal cortex (VLPFC),

112 orbitofrontal cortex (OFC), insular cortex, parahippocampal gyrus, hippocampus, amygdala,

113 fusiform, and ACC. Previous studies provide support for each of those nine a priori ROI (George

114 et al., 2001; Wrase et al., 2002; Tapert et al., 2003; Myrick et al., 2004; Heinz et al., 2007; Ray

115 et al., 2010; Goldstein \& Volkow, 2011; Schacht et al., 2013; Field et al., 2014; Alba-Ferrara et

116 al., 2016; Sawyer et al., 2020). The tenth ROI is the "extended reward and oversight system"

117 (EROS) as described and named in our previous papers (Makris et al., 2008; Sawyer et al.,

118 2017). The EROS ROI is a single large but discontinuous composite ROI that had been created

119 by combining 11 regions (seven of the ROI noted above, all but the VLPFC and fusiform), plus 
120 an additional four: nucleus accumbens, ventral diencephalon, subcallosal cortex, and temporal

121 pole. For each of the ten ROI, we intended to confirm findings from each of the aforementioned

122 studies that had identified abnormal activation by alcohol cues in AUD, and additionally to

123 investigate differences between men and women. We expected lower brain activation in the ALC

124 group in regions involved in facial identity and inhibition of distractor interference, but higher in

125 regions responsible for reward salience.

126 In summary, we investigated brain activation for ten ROI, and for vertex-wise cortical

127 analyses of fixation-regions and task-regions. We examined the accuracy of the participants'

128 memory for the face identities after exposure to attentionally salient pictures to test our

129 hypothesis that alcohol cues would distract the ALC group more than the NC group. We

130 determined how brain regions were activated by the distractor contrasts, how the contrasts

131 differed for ALC and NC groups, and how those abnormalities varied by gender. We

132 hypothesized that attentional biasing would be evident for the ALC group in the form of stronger

133 brain activity contrasts (alcoholic beverage cues compared to nonalcoholic and scrambled

134 stimuli). Regarding gender differences, we made predictions based upon previous work in our

135 laboratory wherein we found that brain regions of $\mathrm{ALCw}$ (compared to $\mathrm{NCw}$ ) were overactive in

136 response to highly charged emotional stimuli (Sawyer et al., 2019). We hypothesized that the

137 ALCw would evidence hyperactivation to emotionally valent stimuli, whereas the activation

138 contrasts for ALCm would be weaker. We also expected to replicate prior results (Marinkovic et

139 al., 2009; Sawyer et al., 2019) showing lower responses in ALCm than NCm. 


\section{2. Materials and Methods}

\subsection{Participants}

$144 \quad$ Participants in this study included 42 abstinent long-term ALC individuals (21 ALCw)

145 and $42 \mathrm{NC}$ controls (21 NCw), with comparable age, education, and IQ (see Table 1 in the

146 Results). Participants were recruited through flyers placed in treatment and after-care facilities,

147 the Boston VA Healthcare System facility, Massachusetts General Hospital, the Boston

148 University School of Medicine, and in public places (e.g., churches, stores), as well as through

149 newspaper and internet advertisements. This study was reviewed and approved by human studies

150 Institutional Review Boards at the affiliated institutions. All participants gave written informed

151 consent prior to participation, and they were compensated for their time.

152 Selection procedures included a telephone interview to determine age, education, health

153 and alcohol and drug use history, including prescription drugs. Participants were right-handed,

154 had normal or corrected-to-normal vision, and spoke English as their first language (or had

155 acquired English as a second language by age five). Current drug use excepting nicotine was

156 cause for exclusion, as were history of alcohol-related liver disease, epilepsy, head trauma

157 resulting in loss of consciousness for 15 minutes or more, HIV, schizophrenia, or metal implants.

\subsection{Neuropsychological Assessment}

160 Neuropsychological testing was conducted at the Department of Veterans Affairs (VA)

161 Boston Healthcare System facility prior to scanning. Participants completed a medical history

162 interview, vision test, handedness questionnaire (Briggs \& Nebes, 1975), and a battery of tests as

163 described below. All subjects were screened using the Hamilton Rating Scale for Depression

164 (Hamilton, 1960) and the Diagnostic Interview Schedule for the DSM-IV (Robins et al., 2000). 
165 The majority of participants also were administered the Wechsler Adult Intelligence Scale

166 (WAIS-III) and the Wechsler Memory Scale (WMS-III) (Wechsler, 1997). Four participants

167 (two ALCw and two ALCm) received the WAIS-IV and WMS-IV (Holdnack \& Drozdick,

168 2010), and WMS-III scores were not obtained from one ALCm. The scores for these participants

169 were adjusted to account for differences in scoring outcomes relative to the earlier versions of

170 the scales. Because craving for the rewarding effects of alcohol is known to serve as a trigger for

171 relapse in those recovering from AUD (Schneider et al., 2001), and alcohol cue exposure in

172 particular is known to be related to relapse (Lubman, 2007), all participants were administered

173 the Penn Alcohol Craving Scale (Flannery et al., 1999) immediately before and approximately

174 two weeks following the scan to assess any changes in alcohol craving patterns.

175

\subsection{Alcohol Screening}

The ALC participants met criteria for alcohol abuse or dependence, and consumed 21 or

178 more alcoholic drinks per week for five or more years. Extent of alcohol use was assessed by

179 calculating Quantity Frequency Index (QFI) scores (Cahalan et al., 1969). QFI scores

180 approximate the number of drinks consumed per day, and take into consideration the amount,

181 type, and frequency of alcohol consumption either over the last six months (NC participants), or

182 over the six months preceding cessation of drinking (ALC participants), and yields an estimate of

183 ounces of ethanol per day. To remove the influence of current alcohol abuse, ALC participants

184 must have been abstinent for at least four weeks before the scan date to be included. The ALC

185 participants did not display symptoms of Korsakoff's Syndrome nor dementia (Oscar-Berman \&

186 Maleki, 2019). Potential NC participants who had consumed 15-20 drinks per week for any

187 length of time or who engaged in binge drinking were disqualified. 


\subsection{Functional Imaging Task}

All participants were given a delayed matching-to-sample memory task (Dolcos \&

191 McCarthy, 2006) in a magnetic resonance imaging (MRI) scanner, whereby they were asked to

192 encode two faces that both had one of three emotional valences: positive, neutral, or negative

193 (Figure 1). The face stimuli were shown in grayscale and were taken from a set of faces used in a

194 previous study (Marinkovic et al., 2009). These faces were displayed simultaneously for three

195 seconds, followed by an asterisk (*) for one second. Subjects were asked to maintain these faces

196 in memory while a colored distractor stimulus was shown. On different trials, the distractor

197 stimulus was either a picture of an alcoholic beverage (alcbev; beer, wine, liquor, or mixed

198 drink), a picture of a nonalcoholic beverage (nonalcbev; water, juice, milk, soda, coffee, tea,

199 etc.), or a scrambled nonsense picture (scrambled). Alcoholic and nonalcoholic beverage pictures

200 were a combination of images used with permission from the Normative Appetitive Picture

201 System (NAPS) (Stritzke et al., 2004), and other previously published works on alcohol cues

202 (Grüsser et al., 2000; Wrase et al., 2002; Myrick et al., 2004). Additional distractor images were

203 modified from digital photographs taken at bars, liquor stores, and convenience stores. The

204 scrambled images were created by inverting half the alcoholic and half the nonalcoholic

205 beverage images and distorting them until they were not recognizable as any particular object,

206 while preserving a match of primary visual characteristics. Each distractor picture was shown for

207 three seconds, followed by an asterisk (*) for one second. Following the distractor picture, a

208 single probe face was shown for two seconds, and the participants were instructed to report

209 whether this face was one of the two faces they had just seen. 
Each trial was 10 seconds in length, and was followed by a variable delay period (with a

211 mean duration of 10 seconds, ranging from 2-22 seconds) during which the subject saw a set of

212 crosshairs (+++) serving as a visual fixation. The task was divided into nine runs, each of which

213 contained 18 trials. There were nine trial types made up of each combination of face valence and

214 distractor type (e.g., positive faces followed by alcohol distractor). Each emotion-distractor

215 combination appeared twice per run. The stimulus order and variable inter-trial intervals were

216 determined using optseq2 (http://surfer.nmr.mgh.harvard.edu/optseq), which optimizes statistical

217 efficiency and hemodynamic response estimate accuracy for event-related experimental designs

218 (Dale, 1999). In total, there were 54 trials for each distractor type (combined across facial

219 expressions) and each face valence (combined across distractor types), for a total of 162 trials

220 across the entire scan. The stimulus faces were balanced to contain $50 \%$ male and $50 \%$ female

221 faces. Within a trial, the two encoded faces and probe face were matched on emotional

222 expression and gender. This way, on match trials the probe facial image was identical to one of

223 the encoded images, and on mismatch trials the facial identity changed but the emotional

224 expression and gender did not.

225 The probe face matched one of the encoded faces on $50 \%$ of the trials, and

226 match/mismatch trials appeared in a randomized order within each run. Responses were made by

227 pressing one of two buttons with the index finger (match) or middle finger (mismatch) of the

228 right hand. Participants were instructed to respond as quickly as possible without sacrificing

229 accuracy. Additionally, participants could immediately correct a response by pressing the

230 opposite button. To ensure the distractor images were viewed by all participants, they were told

231 that it was necessary to pay attention to the pictures shown in between the faces on each trial, as

232 they would be questioned about those images following the scan. 


\subsection{Behavioral Task Analyses}

Responses to the face memory task were analyzed on the first level (individual subjects)

236 using custom Excel templates. Trials were organized by Distractor type, facial Emotion, and

237 Face Gender. Each trial was scored as correct, incorrect, or miss (i.e., no response). When more

238 than one response was made to a trial, the last response type (i.e., yes/match or no/nonmatch)

239 was accepted as the final answer, provided that the final response was at least $200 \mathrm{~ms}$ after the

240 preceding response and no more than $10 \mathrm{~s}$ following the preceding response. When a single

241 response was recorded and the reaction time was less than $200 \mathrm{~ms}$, the trial was scored as a miss.

242 For each participant, a mean overall reaction time (regardless of trial type) was calculated.

243 Reaction times that exceeded three standard deviations from this mean were excluded from

244 reaction time calculations by trial type. Participants' patterns of responses were analyzed for

245 consecutive misses to assure that they remained awake throughout the task. Three separate runs

246 were identified, each in a different participant, wherein greater than five consecutive trials were

247 missed; these runs were excluded from behavioral analyses.

248 Second level (group) effects on percent correct and reaction time (correct trials) were

249 investigated using SPSS Version 17.0 (IBM, Chicago, IL, USA). Repeated-measures analyses of

250 variance (ANOVA) were carried out with between-subjects factors of Group (ALC or NC) and

251 Gender (female participant or male participant) and within-subjects factors of Distractor (alcbev,

252 nonalcbev, or scrambled), Emotion (positive, negative, or neutral), and Face Gender (female face

253 or male face). 


\subsection{Image Acquisition}

Imaging was conducted at the Massachusetts General Hospital's Athinoula A. Martinos

257 Center for Biomedical Imaging in Charlestown, MA. Data were acquired on a 3 Tesla Siemens

258 (Erlangen, Germany) MAGNETOM Trio Tim MRI scanner with a 12-channel head coil. Sagittal

259 T1-weighted MP-RAGE scans $\left(\mathrm{TR}=2530 \mathrm{~ms}, \mathrm{TE}=3.39 \mathrm{~ms}\right.$, flip angle $=7^{\circ}, \mathrm{FOV}=256 \mathrm{~mm}$,

260 slice thickness $=1.33 \mathrm{~mm}$, slices $=128$, matrix $=256 \times 192)$ were collected for all subjects. For

261 most participants, two such volumes were collected and averaged to aid in motion correction. An

262 auto-align localizer was employed to adjust the acquired slices such that they ran parallel to an

263 imaginary plane between the anterior and posterior commissures. Echo planar functional MRI

264 blood oxygen level dependent (BOLD) scans were collected axially with $5 \mathrm{~mm}$ slice thickness

265 and $3.125 \times 3.125 \mathrm{~mm}$ in-plane resolution (64 x 64 matrix), allowing for whole brain coverage

266 (32 interleaved slices, $\mathrm{TR}=2 \mathrm{~s}, \mathrm{TE}=30 \mathrm{~ms}$, flip angle $=90^{\circ}$ ). The event-related design included

26718 trials per run with a total of nine runs. Within each six-minute run, $180 \mathrm{~T}_{2}{ }^{*}$-weighted volumes

268 were collected. Functional volumes were auto-aligned to the anterior/posterior commissure line

269 to ensure a similar slice prescription was employed across participants. Prospective Acquisition

270 Correction (3D-PACE) was applied during collection of the functional volumes to minimize the

271 influence of participants' body motion (Thesen et al., 2000). An IBM ThinkPad (Windows XP)

272 running Presentation version 11.2 (NeuroBehavioral Systems, Albany, CA) software was used

273 for visual presentation of the experimental stimuli and collection of participants' responses.

274 Stimuli were back-projected onto a screen at the back of the scanner bore and were viewed by

275 the participants through a mirror mounted to the head coil. All participants wore earplugs to

276 attenuate scanner noise. 


\subsection{Structural Image Processing}

Structural MPRAGE image analyses were performed for all participant data using the

280 FreeSurfer (version 4.5.0) image analysis suite (http://surfer.nmr.mgh.harvard.edu). A multi-

281 stage cortical surface reconstruction process was run on the two collected T1-weighted MP-

282 RAGE scans (Dale et al., 1999), starting with motion correction, intensity normalization (Sled et

283 al., 1998), Talairach registration (Talairach \& Tournoux, 1988), skull stripping (Ségonne et al.,

284 2004), and segmentation (Fischl et al., 2002) of white matter, gray matter, and ventricles.

285 Subsequently, boundaries were calculated delineating where gray and white matter meet, and

286 where gray matter adjoins cerebrospinal fluid ("pial surfaces") based on maximal shifts in image

287 intensity between tissue types. These boundaries, as well as the subcortical segmentations, were

288 visually inspected on each coronal slice for every subject, and manual interventions (e.g., white

289 matter volume corrections) were made when needed. The surface boundaries were used to

290 generate computationally inflated two-dimensional cortical surface models, which allowed

291 individual subjects to be registered to a spherical atlas by utilizing each subject's cortical folding

292 patterns. This registration was used to align the cortical geometry of all subjects within a group.

293 Creation of these cortical surface models allowed improved data visualization as well as

294 improved accuracy of within-group co-registration relative to an affine morph procedure (Fischl

295 et al., 1999). The cortical surface models were employed in an automated parcellation procedure

296 that divides the surface into subregions based on gyral and sulcal anatomy. The Destrieux atlas

297 parcellation for FreeSurfer (Destrieux et al., 2010) was used to define anatomical ROI in the

298 functional analyses. 


\subsection{Functional Image Processing and Statistical Analyses}

Effects of Group, Gender, Distractor, and Emotion on the BOLD signal were evaluated

using both a whole-brain cluster analysis as well as ROI analyses. Processing of the functional data was performed using the FreeSurfer Functional Analysis Stream (FS-FAST) version 5.3,

SPSS Version 17.0, and Matlab 7.4.0.

\subsubsection{First-Level Functional Analyses}

analysis included motion correction, intensity normalization (Sled et al., 1998), and spatial smoothing with a 5-mm Gaussian convolution kernel at full-width half-maximum. Trials were first combined across runs by distractor-emotional face valence pairs (i.e., alcbev-positive,

311 alcbev-negative, alcbev-neutral, nonalcbev-positive, nonalcbev-negative, nonalcbev-neutral,

312 scrambled-positive, scrambled-negative, scrambled-neutral) and then collapsed across emotional

313 valence. The BOLD response was estimated using a Finite Impulse Response (FIR) model,

314 which allows for estimation of the time course of activity (percent signal change for a given

315 condition) within a vertex or ROI for the entire trial period. For each condition, estimates of

316 signal intensity were calculated for 2 pre-trial and 10 post-trial onset TRs, for a total analysis

317 window of 24 seconds. Motion correction parameters calculated during alignment of the

318 functional images were entered into the analysis as external regressors. Alignment of the T2*-

319 weighted functional images with T1-weighted structural volumes was accomplished through an

320 automated boundary-based registration procedure (Greve \& Fischl, 2009). These automated

321 alignments were manually inspected to ensure accuracy. 
323 between experimental conditions. Three contrasts were used to identify DMN regions (as

324 described below); they were made between distractor types and fixation: (1) alcbev vs. fixation,

325 (2) nonalcbev vs. fixation, and (3) scrambled vs. fixation. Another three contrasts were used to

326 assess cue responsivity: (1) alcbev vs. nonalcbev, (2) alcbev vs. scrambled, (3) nonalcbev vs.

327 scrambled. Analyses of each of these contrasts included removal of prestimulus differences

328 between the contrasted conditions by averaging the first three time points (two pre-trial onset and

329 one post-trial onset) for each condition and subtracting this mean from each time point for that

330 condition. Time points summed for inclusion in each contrast were chosen to reflect peak

331 stimulus-related activity: FIR estimates of hemodynamic responses to the distractors were

332 analyzed using a mean of the five TRs collected during the time period of 2-12 seconds post

333 distractor onset. Since the distractor is shown 4 seconds after the trial onset, the analysis window

334 is 6-16 seconds following trial onset (time points 3 through 8).

\subsubsection{Cortical Surface Cluster Analyses}

337 We investigated cue-related brain activation in two separate cortical brain networks: (1)

338 cue reactivity in DMN regions, and (2) cue reactivity in task-regions. The brain network that was

339 more active during presentation of the fixation cue than during the distractor images was used as

340 our measure of DMN regions (fixation-regions). The network that was more active during the

341 presentation of distractor images than during presentation of the fixation stimulus was used as

342 our measure of the task-regions. In what follows, we first describe masking procedures and

343 analyses we used to separate the networks. 
The $t$-statistic maps for each condition vs. fixation were thresholded at $p<0.05$ vertex-

345 wise and were used to generate binary masks (Figure 2) separating fixation-regions and task-

346 regions, thereby forming the six masks: alcbev greater than (or less than) fixation, nonalcbev

347 greater than (or less than) fixation, scrambled greater than (or less than) fixation. These masks

348 were used to separate the between-distractor analyses (described below).

349 Second-level (group) analyses on cortical regions were accomplished using FS-FAST, a

350 surface-based morphing procedure for intersubject alignment and statistics (Fischl et al., 1999).

351 Group-averaged signal intensities during each experimental condition (alcbev, nonalcbev,

352 scrambled) relative to fixation were calculated using the general linear model in spherical space

353 for cortical regions, and were mapped onto the canonical cortical surface $f$ saverage, generating

354 group-level weighted random-effects $t$-statistic maps masked to include only the cortex.

355 Weighted random effects models were employed to reduce noise by taking into account

356 individual subject variance. A $5 \mathrm{~mm}$ smoothing kernel (full-width half-maximum) was employed

357 for all group and intergroup maps. Cluster correction on maps showing activity for each

358 distractor condition vs. fixation was applied using FS-FAST Monte Carlo simulation with a

359 clusterwise threshold of $p<0.05$ corrected for three spaces (left hemisphere cortical, right

360 hemisphere cortical, and subcortical). Cortical surface cluster regions were identified by the

361 location of each cluster's peak vertex on the cortical surface according to the Desikan-Killiany

362 atlas (Desikan et al., 2006).

363 When we examined brain activation differences between the distractor types, we used

364 three contrasts: alcbev vs. nonalcbev, alcbev vs. scrambled, and nonalcbev vs. scrambled. We

365 investigated each direction of these contrasts separately. For example, brain regions with higher 
366 activation for alcbev than nonalcbev would be analyzed separately from those regions with

367 higher activation for nonalcbev than alcbev.

368 Intergroup comparison $t$-statistic maps were generated using FS-FAST by comparing

369 activation levels of all of the ALC participants with levels of all of the NC participants.

370 Additionally, Group-by-Gender interaction maps for each contrast were calculated.

\subsubsection{Region of Interest Analyses}

The anatomically-defined ROI for the distractor analyses included areas hypothesized $a$

374 priori to be implicated in alcohol craving, distractor interference, and working memory for

375 emotional faces, as described in the Introduction. These were DLPFC, VLPFC, OFC, insular

376 cortex, parahippocampal gyrus, hippocampus, amygdala, fusiform, ACC, and the multi-regional

377 EROS (Makris et al., 2008; Sawyer et al., 2017). Left and right hemisphere regions were

378 analyzed as separate ROI.

Statistical preprocessing and time course visualization of ROI data were performed using

380 scripts written for Matlab version 7.4.0. Signal intensity for each region was averaged across all

381 vertices (for surface-based ROI) or voxels (for volume-based ROI) included in the region for

382 each condition on the individual participant level. To compute percent signal change for each

383 participant within an ROI, signal estimate per condition and time point was divided by the

384 average baseline activity for that participant. Time courses were normalized at the individual

385 subject level for each condition by taking the mean of the first three time points (two pre-trial

386 and one post-trial onset) and subtracting this mean from each time point. Group and Group-by-

387 Gender averages of the normalized time courses were computed for each condition, and were 
388 visualized by plotting the percent signal change for each condition at each time point (i.e., TR) of

389 the trial.

390 For the distractor ROI analyses, percent signal changes of the BOLD signal within each

391 ROI for the time window from 2 to $12 \mathrm{sec}$ after distractor onset were entered as dependent

392 variables into repeated-measures ANOVA models with between-group factors of Group (ALC or

393 NC) and Gender (men or women) and within-subjects factor of Distractor type (alcbev,

394 nonalcbev, or scrambled).

395

396 3. Results

397 3.1 Research Participant Characteristics

398 Table 1 summarizes means, standard deviations, and ranges of participant demographics,

399 drinking variables, and IQ and memory test scores. 


\section{ALCOHOLICS}

$\begin{array}{cccccc}\text { ALC } & \text { ALCw } & \text { ALCm } & \text { NC } & \text { NCw } & \text { NCm } \\ n=42 & n=21 & n=21 & n=42 & n=21 & n=21\end{array}$

$\overline{\operatorname{Age}^{\mathrm{a}}(\text { years) }}$

mean

standard deviation

range

\section{Education $^{\text {b }}$ (years)}

mean

standard deviation

range

\section{WAIS-III Full Scale IQ}

mean

standard deviation

range

\section{WMS-III IMI}

mean
standard deviation
range

\section{WMS-III DMI}

mean

standard deviation

range

\section{Duration of Heavy Drinking ${ }^{\text {cdef }}$}

(years)

mean

standard deviation

range

\section{Quantity Frequency Index ${ }^{\text {cde }}$}

\section{(ounces ethanol/day;}

$\sim$ drinks/day)

mean

standard deviation

range

\section{Length of Sobriety ${ }^{\text {cde }}$ (years)}

$$
\text { mean }
$$

standard deviation

$\begin{array}{cccccc}53.9 & 53.4 & 54.4 & 53.9 & 57.7 & 50.2 \\ 11.0 & 11.4 & 10.8 & 12.4 & 13.6 & 10.1 \\ 26.5-76.7 & 26.5-73.0 & 26.6-76.7 & 25.8-76.9 & 25.8-76.9 & 29.0-69.6\end{array}$

$\begin{array}{cccccc}14.7 & 15.3 & 14.1 & 15.5 & 15.6 & 15.4 \\ 2.0 & 2.0 & 1.9 & 2.0 & 2.3 & 1.6 \\ 12-19 & 12-19 & 12-18 & 12-19 & 12-20 & 12-18\end{array}$

$\begin{array}{cccccc}110.3 & 110.1 & 110.5 & 111.6 & 111.2 & 112.0 \\ 15.0 & 14.2 & 16.0 & 16.3 & 19.3 & 13.1 \\ 72-140 & 72-137 & 81-140 & 79-152 & 79-142 & 90-152\end{array}$

$\begin{array}{cccccc}109.7 & 114.4 & 104.7 & 111.9 & 114.8 & 109.0 \\ 16.6 & 18.3 & 13.4 & 16.9 & 16.4 & 17.4 \\ 63-144 & 63-144 & 82-130 & 80-146 & 84-138 & 80-146\end{array}$

$\begin{array}{cccccc}112.6 & 116.7 & 108.3 & 111.8 & 113.5 & 110.1 \\ 17.3 & 20.4 & 12.5 & 16.0 & 14.9 & 17.2 \\ 52-140 & 52-140 & 86-132 & 83-150 & 83-140 & 84-150\end{array}$

$\begin{array}{cccccc}17.4 & 14.3 & 20.5 & 0.0 & 0.0 & 0.0 \\ 7.7 & 5.2 & 8.5 & 0.0 & 0.0 & 0.0 \\ 5.0-35.0 & 6.0-25.0 & 5.0-35.0 & 0.0-0.0 & 0.0-0.0 & 0.0-0.0\end{array}$

$\begin{array}{cccccc}11.2 & 8.7 & 13.7 & 0.3 & 0.2 & 0.4 \\ 8.8 & 5.8 & 10.5 & 0.6 & 0.5 & 0.7 \\ 2.7-38.4 & 2.7-28.1 & 4.5-38.4 & 0.0-2.6 & 0.0-2.4 & 0.0-2.6\end{array}$

$\begin{array}{llllll}8.3 & 10.6 & 5.9 & 2.1 & 3.6 & 0.5 \\ 10.3 & 11.1 & 8.8 & 6.4 & 8.5 & 1.3\end{array}$


range

Penn Alcohol Craving Scale ${ }^{\text {cde }}$

$\begin{array}{lcccccc}\text { mean } & 3.8 & 3.8 & 3.9 & 1.2 & 1.1 & 1.3 \\ \text { standard deviation } & 4.4 & 5.1 & 3.8 & 2.0 & 2.2 & 1.8 \\ \text { range } & 0-19 & 0-19 & 0-12 & 0-9 & 0-9 & 0-5\end{array}$

\section{Hamilton Rating Scale for}

Depression $^{\mathrm{g}}$

$\begin{array}{lcccccc}\text { mean } & 3.5 & 4.9 & 2.2 & 2.4 & 3.1 & 1.8 \\ \text { standard deviation } & 4.2 & 4.1 & 4.0 & 2.8 & 3.3 & 2.1 \\ \text { range } & 0-18 & 0-17 & 0-18 & 0-12 & 0-12 & 0-8\end{array}$

\section{Table 1. Participant characteristics}

402 Participants Characteristics $(p<0.05):{ }^{\mathrm{a}}$ Control Women $>$ Control Men; ${ }^{\mathrm{b}}$ Control Men $>$ Alcoholic Men;

$403{ }^{\mathrm{c}}$ Alcoholics > Controls; ${ }^{\mathrm{d}}$ Alcoholic Men > Control Men; ${ }^{\mathrm{e}}$ Alcoholic Women > Control Women; ${ }^{\mathrm{f}}$ Alcoholic Men >

404 Alcoholic Women; ${ }^{\mathrm{g}}$ Alcoholic Women > Alcoholic Men. See Results for additional details on number of

405 participants.

406 Abbreviations: WAIS = Wechsler Adult Intelligence Scale; WMS = Wechsler Memory Scale; IMI = Immediate

407 Memory Index; DMI = Delayed Memory Index. Five NCm and two NCw reported being lifetime abstainers, and 408 one $\mathrm{NCw}$ was unable to report an accurate length of sobriety.

The ALC and NC groups did not differ significantly by age. Although the NCw were

411 older than the NCm, controls did not differ significantly from their respective ALC counterparts

412 by age. ALCm had on average one year less education relative to NCm. Groups did not differ

413 significantly on WAIS-III Full Scale IQ scores. While ALCw had higher Hamilton Rating Scale

414 for Depression scores than AUDm, the average scores for all four subgroups (ALCm, ALCw,

$415 \mathrm{NCm}$, and $\mathrm{NCw}$ ) were low (all means below 5, whereas mild depression threshold is 8), so

416 depression likely contributed little to our observed gender differences.

417 By definition, the ALC group had longer durations of heavy drinking than the NC group.

418 The ALCm on average drank heavily for six years more than did the ALCw, and showed a trend 
toward drinking larger average daily quantities (QFI, $\left.F_{1,40}=3.66, p=0.06\right)$. Five NCm and two

420 NCw reported being lifetime abstainers (and as such did not have relevant length of sobriety

421 values). The ALC group reported higher levels of craving for alcohol than the NC group on the

422 PACS administered immediately prior to the scan; ALCm and ALCw did not differ on reported

423 level of pre-scan alcohol craving. Eighty-one of the 84 participants were reached approximately

424 two weeks after their scan date to be reassessed on alcohol craving level. One ALCw, one

425 ALCm, and one NCw could not be reached for follow up assessment on PACS scores. Neither

426 the ALC group nor the NC group displayed an increase in alcohol craving (i.e., a significant

427 change in PACS scores) from the assessment on their scan date to their follow up assessment.

Measures of participant performance on the face memory task were calculated for overall

431 performance and for performance by each Distractor type and facial Emotion. Means, standard

432 deviations, and ranges are reported for percent correct responses and reaction times in Table A1

433 and Table A2, respectively.

A significant Group-by-Gender interaction was found for accuracy $\left(F_{1,80}=6.880, p=\right.$

435 0.01, Figure A1 and Table A1). The significant interaction indicated that the better performance

436 for the ALCw than the ALCm was larger than the difference between NCw and NCm. Accuracy

437 and reaction times did not vary significantly as a function of the Distractor, nor were there any

438 significant interactions of Distractor with Group or Gender (all $p>0.05$ ). The main effect of

439 Emotion was significant for percent correct responses, wherein ALC and NC participants alike

440 performed better on both positive and negative faces relative to neutral faces. Performance on

441 positive and negative faces did not differ significantly. The effect of Emotion on percent correct 
442 responses did not vary as a function of Group or Gender (all $p>0.05$ ). Regarding reaction time

443 and Emotion, participants responded more quickly to positive face trials relative to neutral face

444 trials; this effect did not vary by Group nor by Gender. The main effect of Face Gender on

445 percent correct responses also was significant $\left(F_{1,80}=6.80, p=0.01\right)$, with the overall

446 performance being better for male than for female faces. The effects of Group and Gender on

447 percent correct responses and reaction times for Face Gender were not significant (all $p>0.05)$.

\section{3 fMRI BOLD Effects}

Effects of the distractors on the BOLD signal were assessed using group and intergroup

451 cluster analyses for cerebral cortex, along with a-priori analyses of anatomical ROI that had been

452 implicated by the literature. Below, we report group analyses of fixation contrasts and between-

453 distractor conditions, followed by intergroup analyses of the same contrasts.

\subsubsection{Cortical Cluster Analyses of Distractor Effects}

456 Analyses of task contrasts revealed broadly similar activation patterns for the ALCw,

457 ALCm, NCw, and NCm groups. During fixation, regions involved in the DMN (the anatomical

458 network described in the Introduction) were significantly more active than during the

459 presentation of distractor images. We refer to those more active regions as fixation-regions. As

460 detailed in the Methods, these regions were masked and examined separately for subsequent

461 analyses of contrasts between distractor types. Identical analyses were then performed for the

462 task-regions. Significant clusters for between-distractor contrasts can be seen for fixation-regions

463 first (Figure 3 and Figure A3), and then for task-regions (Figure 4 and Figure A4). 
All four groups had more brain activity (Table A3) in response to alcbev than scrambled

465 distractors in the four main fixation-regions (the anterior and posterior medial hub regions, the

466 temporal parietal junction, and the middle temporal gyrus), while the nonalcbev vs. scrambled

467 contrast was less consistent. The alcbev vs. nonalcbev contrast generally indicated higher

468 activation for the alcbev than nonalcbev. For task-regions, alcbev and nonalcbev elicited higher

469 activation than scrambled in the occipital lobe and adjoining visual areas in temporal and parietal

470 cortex (Table A3, Figure A4).

\section{$472 \quad$ 3.3.2 Distractor Intergroup Cluster Analyses}

473 The pattern of results indicated that $\mathrm{ALCw}$ and $\mathrm{NCm}$ had strong activation contrasts

474 (beverages $>$ scrambled) in visual areas and the medial DMN regions, especially the posterior

475 hub. The ALCw had greater activation contrast than NCw, while ALCm had lower activation

476 contrast than NCm. Table 2 summarizes the regions where significant Group-by-Gender

477 interactions were found for the distractor contrasts, i.e., for alcbev vs. nonalcbev, alcbev vs.

478 scrambled, and nonalcbev vs. scrambled. Table A4 provides all significant Group-by-Gender

479 interactions, and Figure 3, Figure 4, Figure A3, Figure A4 illustrate alcbev vs. scrambled

480 contrasts. In total, we observed 22 clusters where the Group-by-Gender interaction was

481 statistically significant: Seven in fixation-regions and 15 in task-regions. 


\begin{tabular}{llll} 
Fixation Masking Distractor Contrast & Clusters & Annotation (Hemisphere: Number of Clusters) \\
\hline fixation-regions & alcbev > scrambled & 4 & superior frontal (L: 1, R: 2), precuneus (R: 1) \\
fixation-regions & nonalcbev > scrambled & 3 & superior frontal (R: 2), caudal middle frontal (R: 1) \\
task-regions & alcbev > scrambled & 5 & superior parietal (L: 1, R: 2), fusiform (R: 1), lingual (R: 1) \\
task-regions & nonalcbev > scrambled & 4 & superior parietal (L: 1, R: 1), lateral occipital (R: 2) \\
task-regions & scrambled > nonalcbev & 4 & superior frontal (L: 2), rostral middle frontal (L: 2) \\
task-regions & nonalcbev > alcbev & 2 & superior frontal (L: 2) \\
\hline
\end{tabular}

\section{Table 2. Group-by-gender cortical cluster summary}

485 Annotations (using the Desikan-Killiany atlas) are shown for each of the 22 clusters with significant Group-by-

486 Gender interactions in distractor contrasts. The Fixation Masking column refers to the separate analyses conducted

487 for fixation-regions and task-regions as shown in Figure 2. The clusters reported can be understood to span multiple

488 functional regions [71]. That is, they are not limited to a single region, as reported by the maximal vertex or voxel.

489 Abbreviations: alcbev $=$ alcoholic beverages; nonalcbev $=$ nonalcoholic beverages; $\mathrm{L}=$ left hemisphere; $\mathrm{R}=$ right

490 hemisphere. See Table A4 for detailed cluster information. Note: for the fixation-regions, superior frontal and caudal

491 middle frontal are part of the anterior hub; and precuneus is part of the posterior hub.

For the seven clusters in fixation-regions, six were in the anterior hub and one was in the

494 posterior hub. For alcbev > scrambled, we observed one cluster in the posterior hub and three in

495 the anterior hub. For three of these four clusters, ALCw and NCm had the strongest contrasts; in

496 the fourth cluster, ALCw had the strongest contrast, whereas ALCm had the weakest. The

497 remaining three clusters were found for nonalcbev $>$ scrambled. As with the alcbev contrasts,

$498 \mathrm{ALCw}$ and $\mathrm{NCm}$ had the strongest contrasts. All three clusters were in the right hemisphere of

499 the anterior hub. Thus, the overall pattern consistent among the seven clusters was as follows:

500 NCm had stronger contrast (beverage > scrambled) than ALCm, while muted or opposite

501 direction comparison was observed for the women. 
Of the 15 task-regions clusters, two were found for nonalcbev > alcbev (left superior

503 frontal cortex). In both clusters, $\mathrm{NCw}$ had higher activation to nonalcoholic beverages, while

504 NCm had higher activation to alcoholic beverages. In the other 13 clusters, the strongest

505 contrasts were found for $\mathrm{NCm}$ and $\mathrm{ALCw}$. Of those, the significant interactions were found in

506 visual regions where nonalcbev > scrambled, while significant interactions in frontal regions

507 were found for scrambled $>$ nonalcbev.

508 Setting aside gender, analyses of activation levels comparing ALC and NC groups

509 revealed 15 cortical clusters with significantly greater contrast levels for the ALC group than for

510 the NC group (Table A5). Two of the clusters were in the DMN: one alcbev-region

511 (temporoparietal junction), and one nonalcbev-region (posterior hub). The other 13, all alcbev-

512 regions, were in task-regions located throughout the cortex: 7 frontal, 3 temporal, 2 parietal, and

5131 occipital.

\section{$515 \quad$ 3.3.3 Distractor Region of Interest Analyses}

516 Results of ANOVAs examining between-subjects effects of Group and Gender and

517 within-subjects effects of Distractor type on BOLD percent signal change within each ROI are

518 summarized in Table 3. Reported means and standard deviations represent the percent signal

519 change across each ROI (unmasked) for the time period of 2 to 12 seconds post-distractor

520 stimulus onset. These anatomically-defined ROI included regions in EROS areas (Makris et al.,

521 2008; Sawyer et al., 2017), as well as in regions associated with face memory maintenance and

522 distractor interference, as described in the Introduction and Methods.

523 Results from the ROI analyses of distractor effects indicated strong effects of distractor

524 type on neural activation patterns. Specifically, among all participants, significantly higher 
525 responses were observed in several regions (EROS, left DLPFC, left VLPFC, right OFC,

526 bilateral parahippocampal gyrus, bilateral hippocampus, bilateral amygdala, and bilateral

527 fusiform) to both alcbev and nonalcbev relative to scrambled stimuli. The effect was

528 significantly larger for the ALC group than the NC group in the left EROS and left fusiform

529 regions, and in four prefrontal brain areas (left DLPFC, left VLPFC, left OFC, and right OFC).

530 Additionally, in the alcbev vs. nonalcbev contrast, the ALC group showed significantly higher

531 responses than the NC group in the left OFC ROI. Figure 5 shows percent signal change over

532 time for the OFC, VLPFC, fusiform, and ACC activation, to demonstrate representative activity

533 patterns. The left OFC shows the heightened activation for alcbev for the ALC group, the

534 fusiform and VLPFC show higher activation in both groups to both beverage types, and the ACC

535 demonstrates how activation is lower during distractor presentation than during fixation (for both

536 groups and for all three distractor types).

537 Applying a Bonferroni correction accounting for all 25 tests would set a critical value for

538 statistical significance of main effects and Distractor, Group, and Gender interactions at $p<$

539 0.002. Using this threshold, main effects of Distractor would remain significant in left OFC,

540 bilateral parahippocampal gyrus, bilateral hippocampus, right amygdala, and bilateral fusiform

541 gyrus. All main effects of Group and Gender, as well as all interaction effects were $p>0.002$. 


\section{ALCOHOLICS}

\begin{tabular}{|c|c|c|c|c|c|c|c|c|c|}
\hline & $\begin{array}{l}\text { Alcoholic } \\
\text { Beverage } \\
\text { Percent S }\end{array}$ & $\begin{array}{l}\text { Nonalcoholic } \\
\text { Beverage } \\
\text { ignal Change: }\end{array}$ & $\begin{array}{l}\text { Scrambled } \\
\text { Picture } \\
\text { Mean } \pm \text { SD }\end{array}$ & $\begin{array}{c}\text { Distractor } \\
\text { Main Effect } \\
F_{1,41}\end{array}$ & $\begin{array}{l}\text { Alcoholic } \\
\text { Beverage } \\
\text { Percent Si }\end{array}$ & $\begin{array}{l}\text { Nonalcoholic } \\
\text { Beverage } \\
\text { gnal Change: } N\end{array}$ & $\begin{array}{l}\text { Scrambled } \\
\text { Picture } \\
\text { Iean } \pm \mathrm{SD}\end{array}$ & $\begin{array}{c}\text { Distractor } \\
\text { Main Effect } \\
F_{1,41}\end{array}$ & $\begin{array}{c}\text { Distractor } \\
\text { by Group } \\
F_{1,80}\end{array}$ \\
\hline$\overline{\text { EROS }}$ & $.073 \pm .093$ & $.059 \pm .072$ & $.030 \pm .068$ & $8.795 * a b$ & $.047 \pm .089$ & $.051 \pm .085$ & $.040 \pm .063$ & .407 & $3.650^{+}$ \\
\hline EROS LH & $.071 \pm .097$ & $.058 \pm .079$ & $.024 \pm .071$ & $10.567 * a b$ & $.049 \pm .093$ & $.055 \pm .093$ & $.040 \pm .066$ & .580 & $4.217 *$ \\
\hline EROS RH & $.075 \pm .093$ & $.061 \pm .071$ & $.037 \pm .069$ & $7.003 * a$ & $.047 \pm .089$ & $.048 \pm .081$ & $.041 \pm .067$ & .247 & $3.034^{+}$ \\
\hline DLPFC LH & $.060 \pm .111$ & $.049 \pm .103$ & $.017 \pm .085$ & $7.529 * a b$ & $.041 \pm .098$ & $.047 \pm .096$ & $.040 \pm .073$ & .032 & $4.481 *$ \\
\hline DLPFC RH & $.069 \pm .111$ & $.060 \pm .094$ & $.039 \pm .077$ & $3.953+$ & $.034 \pm .097$ & $.035 \pm .093$ & $.039 \pm .077$ & .169 & $3.382^{+}$ \\
\hline VLPFC LH & $.198 \pm .132$ & $.181 \pm .147$ & $.145 \pm .111$ & $11.680 * a b$ & $.157 \pm .115$ & $.182 \pm .129$ & $.154 \pm .097$ & .062 & $5.820 *$ \\
\hline VLPFC RH & $.210 \pm .122$ & $.195 \pm .134$ & $.173 \pm .124$ & $5.387 * \mathrm{a}$ & $.157 \pm .114$ & $.174 \pm .134$ & $.155 \pm .104$ & .039 & $2.925^{+}$ \\
\hline OFC LH & $.125 \pm .130$ & $.090 \pm .135$ & $.057 \pm .107$ & $16.019 * a c$ & $.071 \pm .164$ & $.085 \pm .193$ & $.058 \pm .157$ & .399 & $6.105^{*}$ \\
\hline OFC RH & $.256 \pm .179$ & $.234 \pm .203$ & $.178 \pm .210$ & $10.130 * a b$ & $.184 \pm .224$ & $.195 \pm .249$ & $.177 \pm .199$ & .176 & $5.608 *$ \\
\hline Insula LH & $.104 \pm .105$ & $.096 \pm .091$ & $.069 \pm .077$ & $5.920 * a$ & $.067 \pm .088$ & $.082 \pm .080$ & $.066 \pm .061$ & .001 & $3.275^{+}$ \\
\hline Insula RH & $.098 \pm .097$ & $.082 \pm .091$ & $.068 \pm .073$ & $4.084 * a$ & .088 & $.068 \pm .075$ & $.059 \pm .066$ & .025 & 2.109 \\
\hline Parahippocampal LH & $.126 \pm .149$ & $.125 \pm .098$ & $.052 \pm .101$ & 12.216*ab & $.130 \pm .136$ & $.136 \pm .141$ & $.083 \pm .100$ & $4.915 * a b$ & 0.894 \\
\hline Parahippocampal RH & $.156 \pm .140$ & $.136 \pm .093$ & $.083 \pm .090$ & $12.220 * a b$ & $.160 \pm .119$ & $.167 \pm .126$ & $.109 \pm .090$ & $9.013 * a b$ & 0.743 \\
\hline Hippocampus LH & $.085 \pm .131$ & $.077 \pm .096$ & $.021 \pm .094$ & $13.908 * a b$ & $.050 \pm .108$ & $.054 \pm .105$ & $.012 \pm .090$ & $7.179 * a b$ & 1.394 \\
\hline Hippocampus RH & $.088 \pm .120$ & $.066 \pm .083$ & $.024 \pm .088$ & $11.669 * a b$ & $.077 \pm .106$ & $.074 \pm .088$ & $.038 \pm .085$ & $7.955 * a b$ & 1.134 \\
\hline Amygdala LH & $.093 \pm .188$ & $.098 \pm .154$ & $.041 \pm .154$ & $6.019 * a b$ & $.094 \pm .194$ & $.095 \pm .203$ & $.064 \pm .137$ & 2.207 & 0.581 \\
\hline Amygdala RH & $.105 \pm .140$ & $.089 \pm .126$ & $.027 \pm .134$ & $14.976 * a b$ & $.121 \pm .175$ & $.118 \pm .160$ & $.085 \pm .130$ & $4.389 * \mathrm{a}$ & 2.519 \\
\hline ACC LH & $-.059 \pm .119$ & $-.080 \pm .094$ & $-.095 \pm .106$ & $5.019 * a$ & $-.092 \pm .109$ & $-.088 \pm .116$ & $-.089 \pm .105$ & .036 & $3.195^{+}$ \\
\hline ACC RH & $-.056 \pm .119$ & $-.068 \pm .102$ & $-.080 \pm .111$ & 2.026 & $-.085 \pm .107$ & $-.089 \pm .100$ & $-.085 \pm .097$ & 0.000 & 1.179 \\
\hline Fusiform LH & $.758 \pm .258$ & $.743 \pm .207$ & $.553 \pm .120$ & 65.492*ab & $.705 \pm .285$ & $.724 \pm .294$ & $.579 \pm .259$ & $22.579 * a b$ & $4.803 *$ \\
\hline Fusiform RH & $.855 \pm .303$ & $.851 \pm .234$ & $.669 \pm .229$ & $49.514 * a b$ & $.823 \pm .292$ & $.845 \pm .301$ & $.694 \pm .253$ & $49.438 * a b$ & $3.322^{+}$ \\
\hline
\end{tabular}

\section{Table 3. Percent signal change for each distractor type by group, for a-priori regions}

544 Distractor by Group F-values are reported from a full factorial ANOVA model with between-groups factors of

545 Group and Gender and within-groups factor of Distractor type. Distractor main effect F-values within each group are

546 reported from ANOVA model including within-subjects factor of Distractor type. Abbreviations: EROS = Extended

547 Reward and Oversight System; DLPFC = Dorsolateral Prefrontal Cortex; VLPFC = Ventrolateral Prefrontal Cortex;

548 OFC $=$ Orbitofrontal Cortex $;$ ACC $=$ Anterior Cingulate Cortex LH $=$ Left Hemisphere RH $=$ Right Hemisphere

549 a. Alcoholic Beverage $>$ Scrambled Picture, $p<.05$

550 b. Nonalcoholic Beverage $>$ Scrambled Picture, $p<.05$

551 c. Alcoholic Beverage $>$ Nonalcoholic Beverage, $p<.05$

$552 * p<.05$

$553+.05<p<.10$ 


\section{Discussion}

\subsection{Behavioral Responses to Probe Face and Distractor Cues}

In this study, ALC and NC participants alike were able to use emotional face valence

558 information to improve face memory, as assessed by a DMTS task (LeDoux, 1996; Dolcos et al.,

559 2005; Dolcos \& McCarthy, 2006). This was evidenced by better memory performance (i.e.,

560 higher accuracy) on positive and negative faces, and faster reaction times to positive faces, than

561 neutral faces. Because it has been shown that alcoholism is associated with impaired emotional

562 perception, and specifically impaired emotional face decoding (Oscar-Berman et al., 1990;

563 Philippot et al., 1999; Clark et al., 2007; Marinkovic et al., 2009; Hoffman et al., 2019; Lewis et

564 al., 2019), we had postulated that normal enhancement of memory by emotional content (in this

565 case, emotional facial expressions) would not be as strong in the ALC group. In those studies,

566 the ALC groups were mostly or exclusively men. However, our results suggest that the ability to

567 use emotional information to aid face memory implicitly may be relatively preserved in AUDw.

568 A significant Group-by-Gender interaction was observed on recognition accuracy as shown in

569 Figure A1 and Table A1. Although the Group-by-Gender interaction for reaction times was not

570 significant (Figure A2 and Table A2), the pattern is congruent with the accuracy data. The higher

571 accuracy of the $\mathrm{ALCw}$ ( $\sim 6$ percentage points better than $\mathrm{ALCm}$ or $\mathrm{NCw})$ may reflect their

572 greater focus and sensitivity to emotional faces and resistance to distraction or underlying

573 differences in personality or motivation (Mosher Ruiz et al., 2017).

$574 \quad$ We had postulated that the ALC group would show more recognition errors after alcohol

575 distractors (relative to other distractor types), whereas the NC group would not. However, we

576 found that regardless of the group, the distractor type did not substantially influence accuracy or

577 reaction time. Although we also had expected performance by the ALC group to be impaired by 
578 alcoholic beverage cues, a significant interaction between group and distractor type was not

579 evident. We did not find an attentional bias effect: The alcoholic beverage distractors (relative to

580 nonalcoholic beverage or scrambled picture distractors) did not disproportionately decrease the

581 number of correctly recalled faces among either the ALC or the NC groups. A review by Field

582 and Cox (2008) suggested that the strength of the bias among ALC groups depends on drinking

583 history. Interestingly, Loeber and colleagues (2009) found that reduced attentional biasing to

584 alcohol cues was associated with longer durations of heavy drinking, and our sample also had

585 long durations. Combining participants with variable drinking histories might have masked the

586 attentional biasing effect. However, measures of neural activity may be more sensitive than

587 behavioral measures to changes associated with long-term heavy drinking.

\subsection{Distractor fMRI Contrasts}

The fMRI contrasts revealed broadly similar patterns of brain activity among the four

591 groups, for both fixation-regions and task-regions. In both brain networks, the beverages (alcbev

592 and nonalcbev) elicited higher activation than the scrambled distractors. This amounts to internal

593 replications of our present fMRI results, with four independent samples (ALCm, ALCw, NCm,

594 and $\mathrm{NCw}$ ) revealing the same fixation-regions, same task-regions, and with mostly the same

595 direction of effects for task contrasts within those regions. The results reflect the existence,

596 location, and extent of the DMN (Buckner \& DiNicola, 2019; Uddin et al., 2019), and also

597 indicate that beverage pictures elicit higher activation than scrambled pictures in the DMN.

598 Moreover, our results indicate that DMN regions are sensitive to the informational content of

599 visual stimuli. In task-regions, the occipital lobe, along with adjoining visual areas in temporal

600 and parietal cortex, were clearly more activated by beverage stimuli than by scrambled images. 
601 The results further suggest that content processing is not solely performed by those visual

602 regions, because activation to beverage cues was identified in middle to posterior cingulate

603 regions, which are involved in a multitude of cognitive functions (Heilbronner \& Hayden, 2016;

604 Yeo et al., 2016).

605

\subsection{Gender Differences}

The present research provides further evidence for the importance of considering gender

608 when exploring effects of alcoholism on the brain (Mann et al., 2005; Ruiz et al., 2013). Many

609 factors contribute to the observed differences in function for abnormalities identified comparing

610 ALCm with NCm vs ALCw with NCw.

611 Cortical group-level cluster analyses revealed significant Group-by-Gender interaction

612 effects in 22 clusters. The general pattern of those findings indicated that ALCm had lower

613 activation contrasts than NCm, while $\mathrm{ALCw}$ had higher activation contrasts than $\mathrm{NCw}$. This

614 pattern was observed primarily in contrasts between beverage and scrambled distractor

615 conditions, and they were found in the two core medial DMN regions, as well as in visual

616 association cortices. A similar pattern of results was found in a previous report (Sawyer et al.,

617 2019) in which emotional vs. neutral image contrasts were lower in ALCm than NCm, and

618 stronger in ALCw than NCw. The lower brain reactivity for ALCm, and higher for ALCw,

619 highlighted gender effects, suggesting possible differences in the underlying basis for

620 development of AUD. Of note, the results from other modalities also have indicated similar

621 directions of the $\mathrm{MMRI}$ effects, with $\mathrm{ALCw}$ having larger reward regions than $\mathrm{NCw}$, and higher

622 fractional anisotropy than $\mathrm{NCw}$, as compared to the smaller regions and lower fractional

623 anisotropy found for ALCm than NCm (Sawyer et al., 2017, 2018). 
625 could be reflective of other gender differences observed in conjunction with AUD. The role of

626 these regions in internal monitoring could relate to differences in pre-existing risk factors (Ruiz

627 \& Oscar-Berman, 2015; Brighton et al., 2016; Mosher Ruiz et al., 2017), or could represent

628 differential consequences of alcohol abuse (Merrill \& Read, 2010). A similar pattern of group

629 differences was identified in cortical regions associated with visual processing. That is, the

630 results could represent a more fundamental impact that is not regionally-specific. In the present

631 study, effects of both increased activation in reward regions and decreased deactivation in DMN

632 regions in response to alcohol pictures were strongest among $\mathrm{ALCw}$ in particular. One reason

633 stronger alcohol cue-specific responses were observed among ALCw could be related to gender-

634 based differences in physiological responses to alcohol cues (Rubio et al., 2013). Aligned with

635 this, larger responses to alcohol cues by female social drinkers relative to male social drinkers

636 have been reported in superior and middle frontal gyri (Seo et al., 2011).

637 Another explanation for greater effects of alcohol cues among women than men could be

638 related to depression (Saraceno et al., 2012). Symptoms of depression among non-treatment

639 seeking heavy drinkers were reported to be correlated with increased activation in response to

640 alcohol cue exposure in the insula, cingulate, ventral tegmentum, striatum, and thalamus

641 (Feldstein Ewing et al., 2010). Because ALCw tend to experience depression and anxiety

642 symptoms (Benishek et al., 1992; Schulte et al., 2009), we expected to see higher responses to

643 alcohol cues among ALCw than ALCm in these regions. Indeed, in our sample, ALCw had

644 higher Hamilton Rating Scale for Depression scores than did ALCm, although the scores for men 645 and women were low. 
647

648

650

651

652

653

654

655

656

657

658

660

661

662

663 observed in other addictions and neuropsychiatric conditions (Broyd et al., 2009; Bednarski et

664

665

666 667 processed, or the way in which it is integrated (Sormaz et al., 2018). Lower activation of the

668 anterior hub specifically has been associated with dynamic attention allocation during task

669

\subsection{Group Differences}

In addition to significant gender interactions, we identified regions with differences between the ALC and NC groups. For the simple comparisons of the ALC group with the NC group, cluster analyses showed that the ALC group had higher activation in 2 fixation-regions and 13 task-regions (Table A5). Differences in the posterior hub were identified in regions with stronger activation to nonalcoholic beverages than to scrambled images, while the other clusters had stronger activation to alcoholic beverages. The higher contrasts observed for the ALC group indicate a processing bias toward beverage cues across fixation- and task-regions. Further, the fact that this cue-sensitivity is not isolated to a single region in the brain likely reflects a widespread divergence in emotional and cognitive activity.

\subsection{Brain Responsivity in Fixation-Regions (Default Network)}

Compared to the NC group, cluster analyses showed that the ALC group, and the ALCw in particular, had stronger contrasts in the anterior and posterior hubs, along with the temporoparietal junction. The results for ROI that include DMN regions also support the finding of contrast dampening in response to alcohol cues. Abnormal DMN functioning has been al., 2011; Zhou et al., 2020). In AUD, abnormal functional connectivity among DMN regions has been reported (Chanraud et al., 2011). ALCw in particular had stronger contrasts for both the anterior and posterior hubs, an abnormality which could indicate a limitation in the level of detail anterior hub specifically has been associated with dynamic attention allocation during task executions (Koshino et al., 2011), suggesting that reduced deactivation of this region during 
670 viewing of alcoholic beverage pictures among alcoholics could be associated with failure to

671 reallocate attention back to the task when the alcohol distractors were presented.

672

\subsection{Brain Responsivity in Regions of Interest}

674 The ROI analysis of the OFC provides evidence for reward-specific processing in the

675 ALC group. In particular, reward-specific processing refers to their higher activation from the

676 contrast between alcbev and nonalcbev. Several studies have reported enhanced OFC activation

677 to alcohol cues (Wrase et al., 2002; Myrick et al., 2008; Ray et al., 2010; Shields \& Gremel,

678 2020), and research has established the role of the OFC in alcohol and drug addiction more

679 generally (Goldstein \& Volkow, 2002). The OFC activity may be particularly important for

680 preoccupation and anticipation stages of the addiction cycle (Koob \& Volkow, 2010).

681 Additionally, activity in this region has been shown to correlate with subjective craving ratings

682 of viewed alcohol cues (Myrick et al., 2004), and further correlated with relapse risk (Reinhard

683 et al., 2015).

684 In many ROI, differences in activation levels among beverage distractor conditions

685 (alcoholic and nonalcoholic) were larger relative to scrambled pictures in the ALC group than in

686 the NC group. The higher responsivity of the ALC group to alcoholic beverages supports our

687 hypothesis of greater attentional bias in the form of stronger alcbev vs. nonalcbev activity

688 contrasts, but we did not expect the ALC group to have greater activation than the NC group to

689 nonalcoholic beverages relative to scrambled cues. One explanation for this result is that many of

690 the nonalcoholic beverages contain caffeine or sugar (e.g., coffee, tea, soda), which, like

691 alcoholic beverages, also stimulate reward-network activity (Garber \& Lustig, 2011). As was

692 suggested in an earlier meta-analysis (Field et al., 2009), the attentional bias for caffeine-related 
693 cues may correlate more strongly with subjective craving than for alcohol-related cues.

694 Moreover, craving for both caffeine and alcohol utilize similar neural circuits as are used for

695 processing alcohol reward (Kunz et al., 2008), as do the effects of sugar-related reward (Avena

696 et al., 2008; Volkow et al., 2013).

The regions that responded more to beverage cues relative to the scrambled pictures were

698 the total EROS and many of its subcomponents (DLPFC, VLPFC, OFC, parahippocampal gyrus,

699 hippocampus, amygdala) and fusiform. In all of the regions where an interaction of Distractor

700 type and Group was identified, the distractor effect was found to be significant among the ALC

701 group, but not among controls. In the EROS, DLPFC, VLPFC, and OFC, the distractor effect in

702 the ALC group was driven by greater activity during both alcoholic and nonalcoholic beverage

703 pictures relative to scrambled pictures. In the OFC, however, the alcohol distractors elicited more

704 activity in the ALC group than did the nonalcoholic beverages or the scrambled pictures. Thus,

705 the strongest ROI effect specific to processing alcohol cues was observed in the OFC. Crucially,

706 this effect was observed only in the ALC group and not in the NC group (i.e., for controls, no

707 ROI were identified where alcohol distractor pictures elicited more activity than did nonalcoholic

708 beverages; see Table 3). The OFC is believed to play a major role in craving and reward function

709 (Koob \& Volkow, 2010).

710 Responses to alcohol and nonalcoholic beverage pictures in the fusiform gyrus were

711 strong among the ALC and NC groups (Figure 5D), as expected, given the fusiform's role in

712 visual object recognition (Pourtois et al., 2009). We hypothesized further that the ALC group's

713 decreased BOLD signal in the fusiform gyrus in response to alcohol cues would provide

714 evidence for stronger distractor interference with face memory maintenance. However, our

715 results showed that any diminishment of the BOLD signal in the fusiform was far outweighed by 
716 its initially higher responses to the alcohol cues. While reductions in fusiform response across

717 the time window of distractor analysis are apparent in the activation time course, an alcohol-

718 specific decrement in the ALC group was not clear. The fact that BOLD responses in the

719 fusiform ROI were consistently lower for scrambled pictures relative to alcohol and nonalcoholic

720 beverage pictures in both the ALC and NC groups suggests that the differences in visual

721 processing demands between conditions may have overridden any potential reduction in the

722 BOLD signal as a result of distractor interference.

723 We hypothesized that a failure to inhibit distractor interference in response to alcohol

724 cues would be associated with lower activity in VLPFC, given this region's role in inhibition of

725 task-irrelevant distracting stimuli (Thompson-Schill et al., 2002; Aron et al., 2004). However,

726 our ROI results showed similarly increased activation of this region for both alcohol and

727 nonalcoholic beverage cues relative to scrambled pictures. This finding suggests that rather than

728 a failure of VLPFC to inhibit distracting stimuli, the higher activity in this region might result

729 from the overriding demand of emotional and reward salience of the alcohol cues. Alternatively,

730 the VLPFC could be involved in inhibition regardless of the distractor type employed during the

731 DMTS delay.

732

$733 \quad 4.7$ Limitations

734 It is not clear to what degree the abnormalities we observed result from or predate heavy

735 drinking. The mean abstinence period for the ALC group was 8.3 years, and since the NC group

736 did not have an 'abstinence period,' we could not covary for sobriety. Still, our AUD cohort had

737 drinking history values representative of the national population (World Health Organization,

738 2019), which thereby improves the generalizability of our results. Sobriety in our subject cohort 
739 points to how persistent the processing deficits in AUD populations are, and how short- and

740 long-term abstinence may have different paths of recovery for men and women (Fama et al.,

741 2020). Nonetheless, our findings illustrate how critical it is to pursue research examining gender

742 differences regarding attentional bias towards reward-related stimuli and pathological alcohol

743 consumption.

744 In conjunction with the multiple-comparison cluster correction procedures employed, the

745 significance level we used $(p<.05)$ has been shown to have higher false-positive rates than

746 expected (Eklund et al., 2016). However, stricter thresholds would increase the chance of false-

747 negative errors, and the significance level we used allows the size of the gender effects to be

748 highlighted. Although we report cluster labels by the location of the peak voxel or vertex, the

749 clusters reported can be understood to span multiple functional regions (Woo et al., 2014). That

750 is, they are not limited to a single region, as reported by the maximal vertex or voxel.

751 Finally, our analyses did not include factors such as cigarette smoking, body mass index,

752 and hormone therapy (Luhar et al., 2013; Oscar-Berman et al., 2014), which could possibly

753 influence alcohol cue processing, reward, and DMN activity.

\section{5. Conclusions}

756 Compared to the NC group, the ALC group had stronger activation for DMN regions, and

757 overactivated reward regions during alcohol cue distraction. This suggests that attentional

758 capture is not limited to reward regions, but also includes DMN regions. If so, the DMN has a

759 role in processing salient aspects of addictive substances.

760 The present study showed that alcohol cue distractors have powerful effects on reward-

761 related regions of the brain, even in the absence of impaired performance when alcohol cues are 
762 employed as distracting stimuli. We also demonstrated that the increased responses in reward

763 regions are accompanied by dampened DMN activity during the presentation of alcohol cues.

764 Our results suggest that these effects are strongest among ALCw, and provide evidence for

765 dimorphic patterns of responses to alcohol cues between ALCm and ALCw.

766

767 Acknowledgements

768 This work was supported by funds from the US Department of Veterans Affairs Clinical

769 Science Research and Development grant I01CX000326; National Institute on Alcohol Abuse

770 and Alcoholism (NIAAA) of the National Institutes of Health US Department of Health and

771 Human Services under Award Numbers R01AA007112, R01AA016624, K05AA00219, and

772 K01AA13402; and shared instrumentation grants 1S10RR023401, 1S10RR019307, and

773 1S10RR023043 from the National Center for Research Resources (now National Center for

774 Advancing Translational Sciences) at the Athinoula A. Martinos Center, Massachusetts General

775 Hospital. Alcoholic and nonalcoholic beverage pictures were a combination of images used with

776 permission from the Normative Appetitive Picture System (NAPS) (Stritzke et al., 2004), and

777 other previously published works on alcohol cues (Wrase et al., 2002; Myrick et al., 2004). The

778 authors thank Elinor Artsy, Sheeva Azma, Anne-Mette Guldberg, Zoe Gravitz, Doug Greve,

779 Steve Lehar, Diane Merritt, Alan Poey, Elizabeth Rickenbacher, Trinity Urban, Maria Valmas,

780 and Robert Zondervan for assistance with consultation, manuscript preparation and recruitment,

781 assessment, preparing testing materials, and neuroimaging of the research participants. Finally,

782 we would like to acknowledge the role of the research participants for making this study

783 possible. The content is solely the responsibility of the authors and does not necessarily represent 
784 the official views of the National Institutes of Health, the U.S. Department of Veterans Affairs,

785 or the United States Government.

\section{$787 \quad$ Figures}

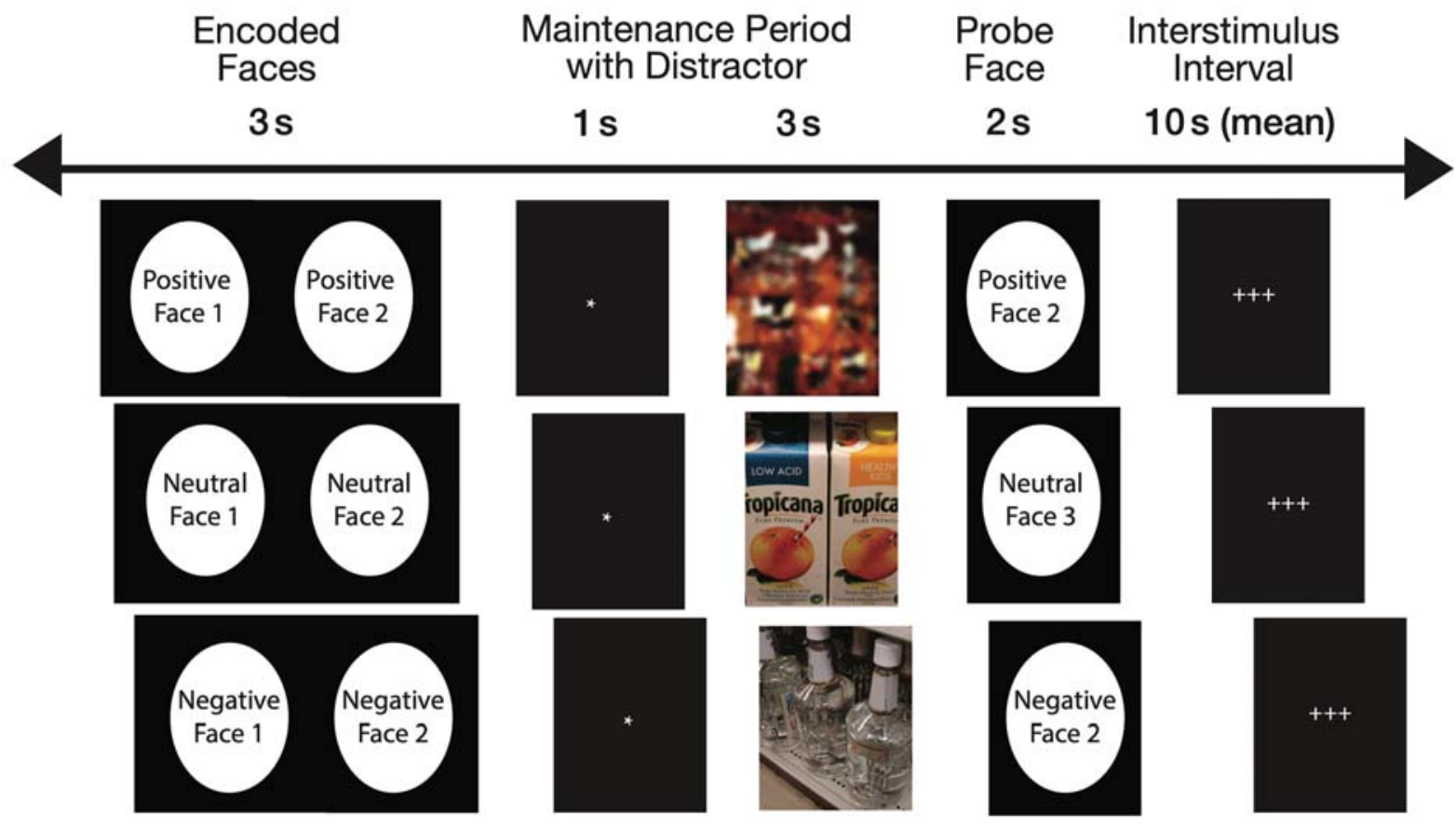

790 Figure 1. Task presented during functional neuroimaging

791 Two faces were presented simultaneously for three seconds, followed by an asterisk for one second. Next, a

792 distractor was presented for three seconds. The probe face immediately followed, during which the subjects had

793 been trained to respond with a button press with either their index or middle finger to indicate whether the probe

794 face matched the encoded face. Three crosses served as the inter-trial interval, which lasted from 2 to 30 seconds

795 (mean 10 seconds). A total of 162 trials were presented. While the faces in this figure have been blurred to mask the

796 identities of the individuals, the research participants saw the original unblurred photographs. 


\section{A. Fixation Masking}

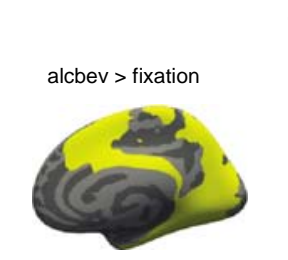

Task Regions
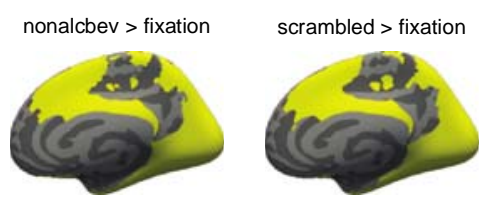

B. Distractor Masking

\section{Task Regions}
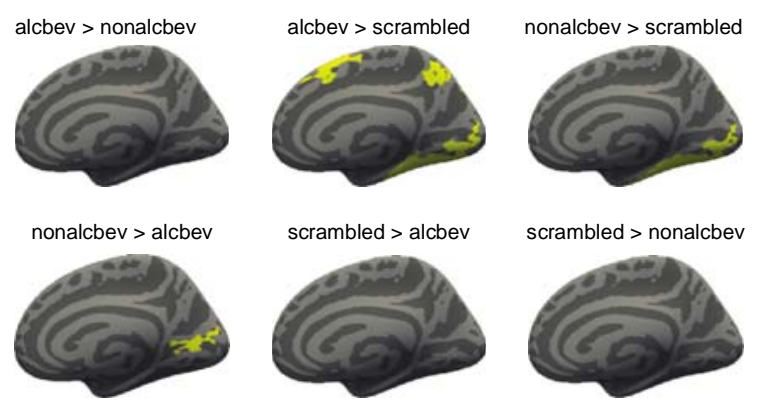

scrambled $>$ nonalcbev

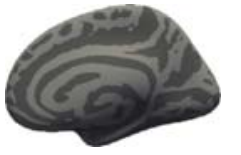

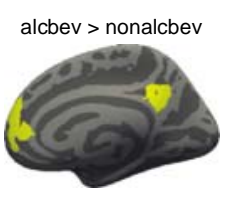

Fixation Regions

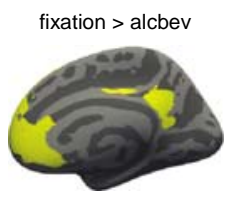

\section{Fixation Regions}
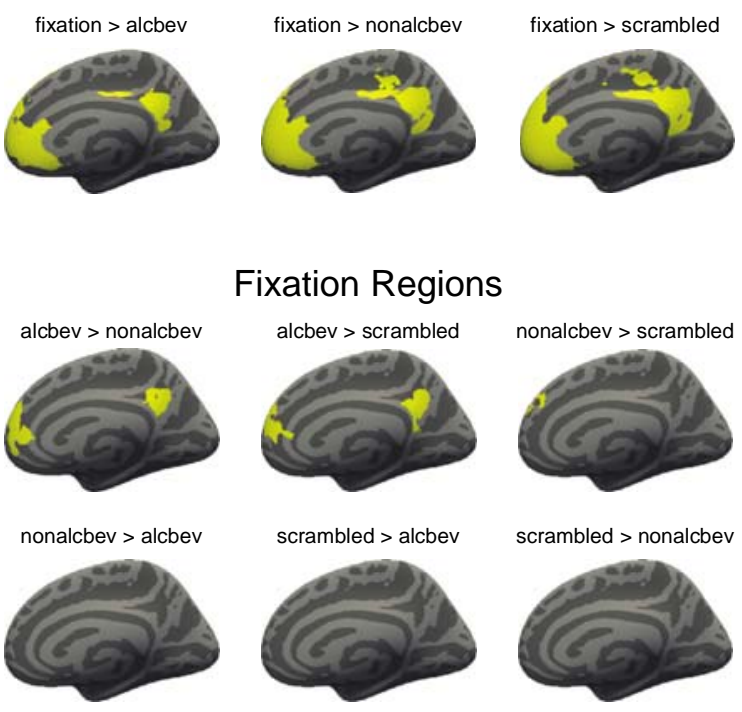

nonalcbev > scrambled
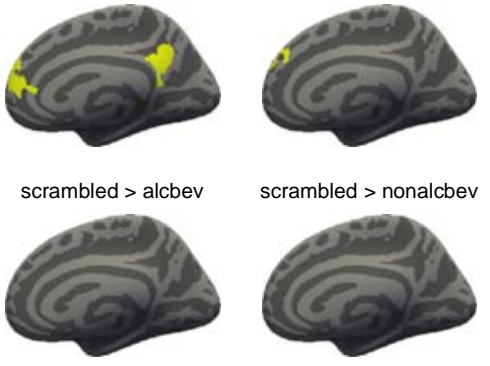

Figure 2. Analyses conducted with masked fMRI data for cortex

800 The top set of brains represents analyses conducted for cortical regions in which network masking (for task and

801 fixation-regions) were performed. The bottom set of brains represents analyses conducted for cortical regions in

802 which distractor masking (for task and fixation-regions) were performed.

803

804 


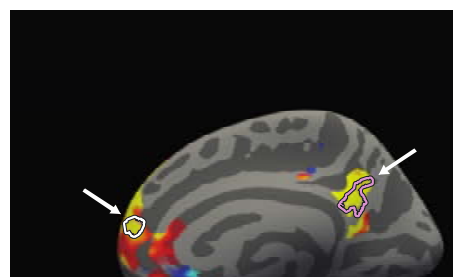

$\mathrm{ALC}_{\mathrm{w}}$

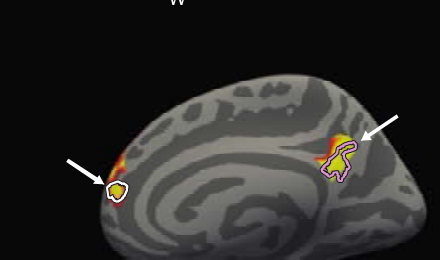

$\mathrm{ALC}_{\mathrm{w}}-\mathrm{NC}_{\mathrm{w}}$

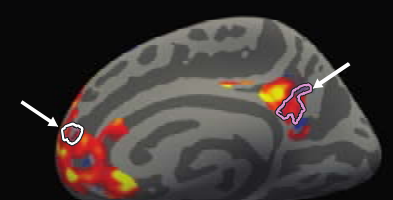

$\mathrm{NC}_{\mathrm{w}}$

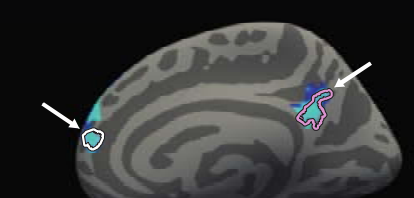

$\mathrm{ALC}_{\mathrm{M}}-\mathrm{ALC}_{\mathrm{W}}$

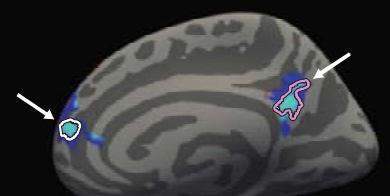

Group by Gender

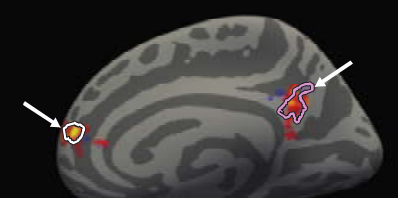

$\mathrm{NC}_{\mathrm{M}}-\mathrm{NC}_{\mathrm{W}}$
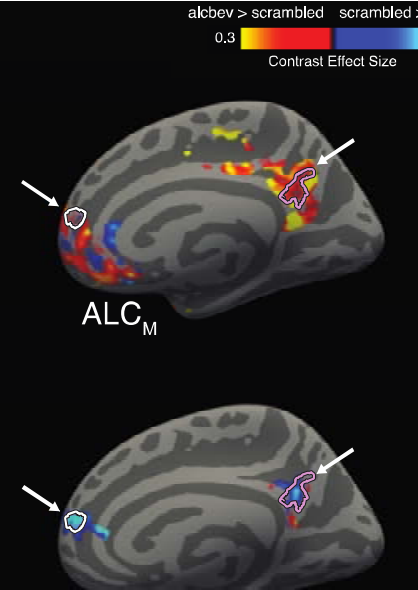

$\mathrm{ALC}_{\mathrm{M}}-\mathrm{NC}_{\mathrm{M}}$

Figure 3. Cortical clusters significant for Group-by-Gender interactions for the alcbev vs.

\section{7 scrambled contrast within fixation-regions (right medial view)}

808 A significant Group-by-Gender interaction revealed several clusters (see Table A4), two of which are indicated by

809 arrows on the medial surface of the right hemisphere, with cluster outlines overlaid on contrast values between

810 alcbev and scrambled distractors. Group mean contrast values (for alcbev vs. scrambled within fixation-regions) are

811 displayed in the four brain images located in the corners of the figure, and group comparisons are indicated by

812 minus signs. Abbreviations: $\mathrm{ALCm}=$ Alcoholic men; $\mathrm{ALCw}=$ Alcoholic women; $\mathrm{NCm}=$ Nonalcoholic men; $\mathrm{NCw}$

$813=$ Nonalcoholic women. 

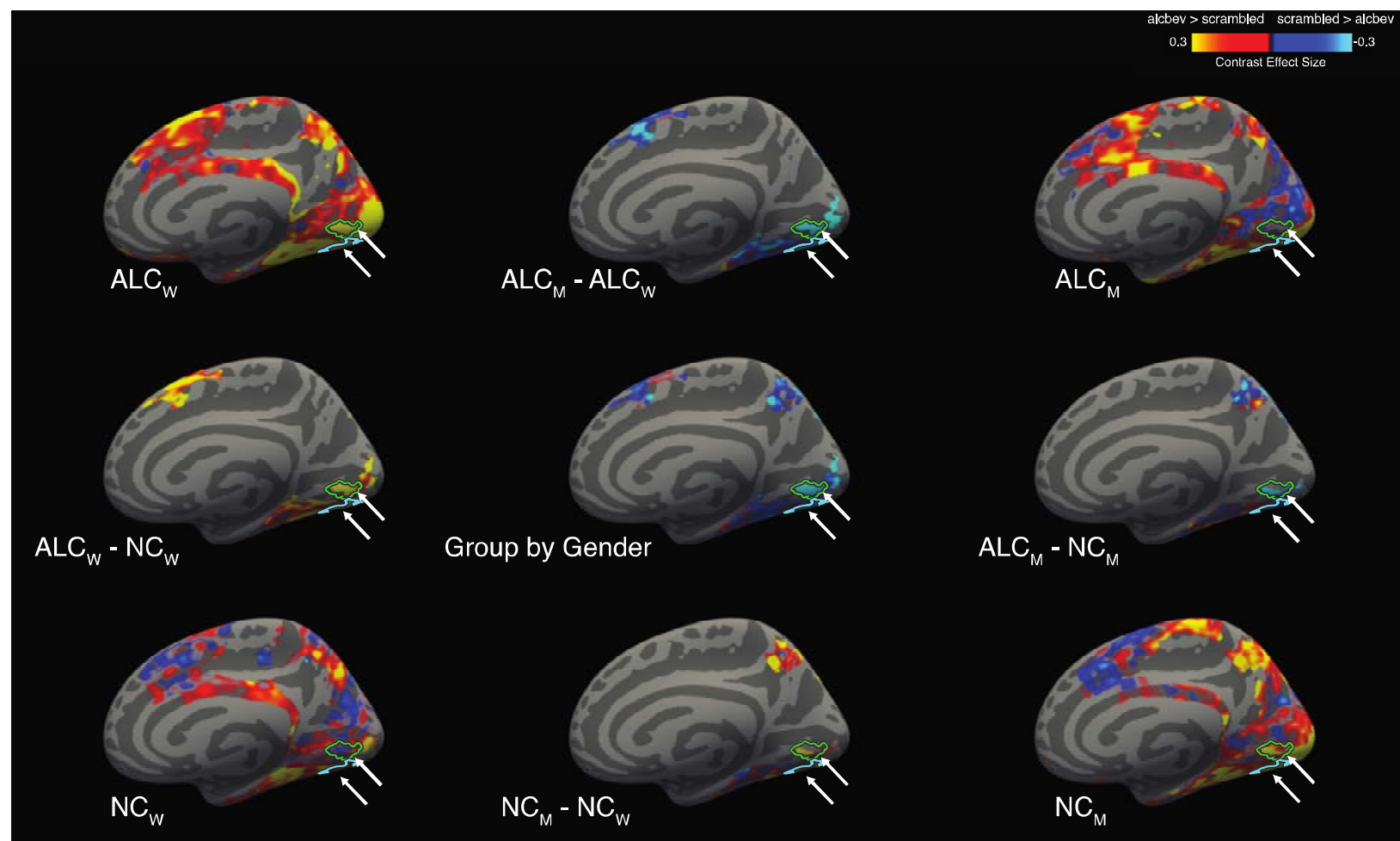

Figure 4. Cortical clusters significant for Group-by-Gender interactions, for the alcbev vs.

\section{1 scrambled contrast within task-regions (right medial view)}

822 A significant Group-by-Gender interaction revealed several clusters (see Table A4), two of which are indicated by

823 arrows on the medial surface of the right hemisphere, with cluster outlines overlaid on contrast values between

824 alcbev and scrambled distractors. Group mean contrast values (for alcbev vs. scrambled within task-regions) are

825 displayed in the four brain images located in the corners of the figure, and group comparisons are indicated by

826 minus signs. Abbreviations: $\mathrm{ALCm}=$ Alcoholic men; $\mathrm{ALCw}=$ Alcoholic women; $\mathrm{NCm}=$ Nonalcoholic men; $\mathrm{NCw}$

$827=$ Nonalcoholic women. 
A. Left OFC

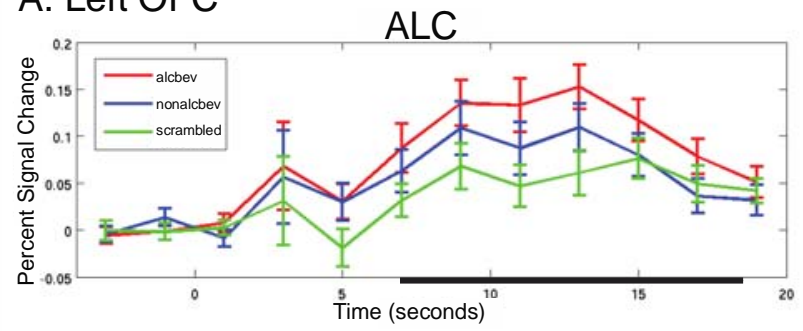

NC

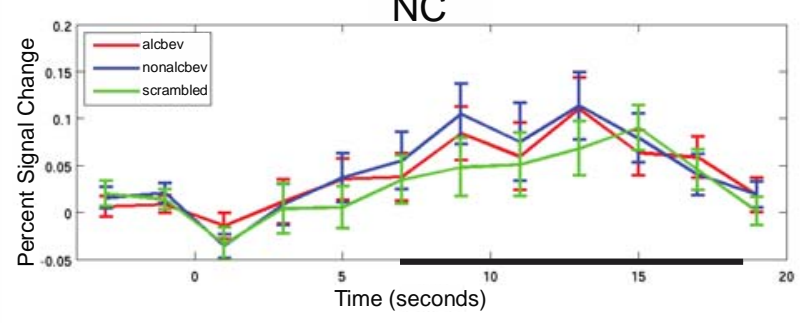

C. Left ACC

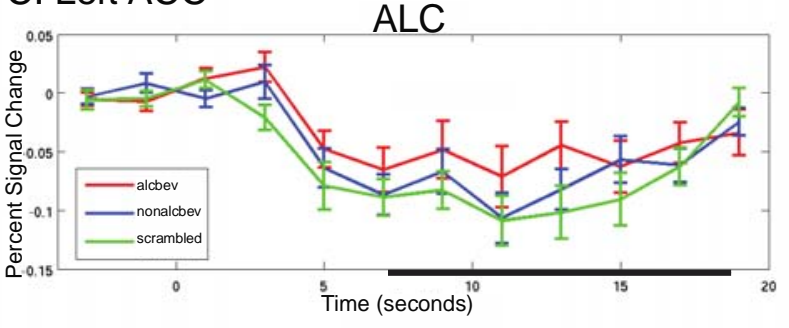

NC

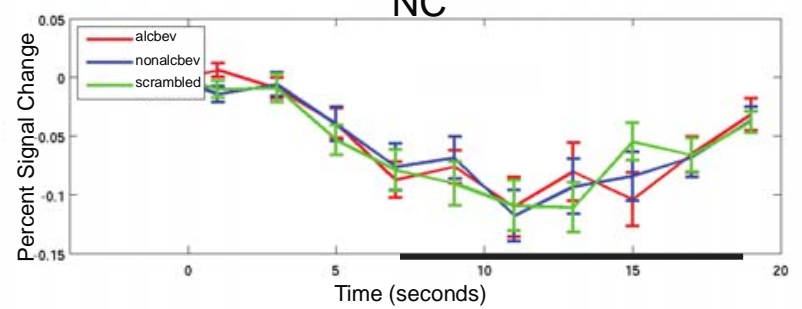

B. Left VLPFC

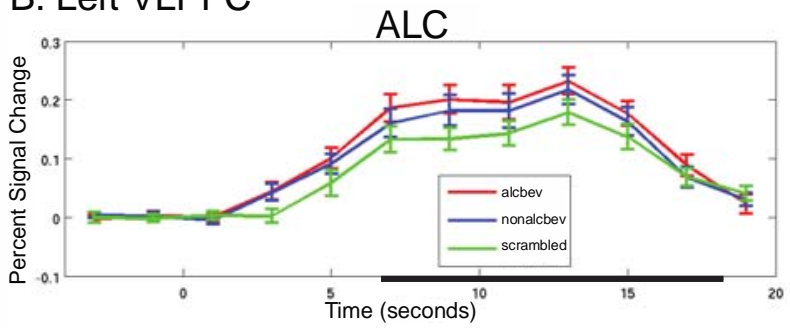

NC

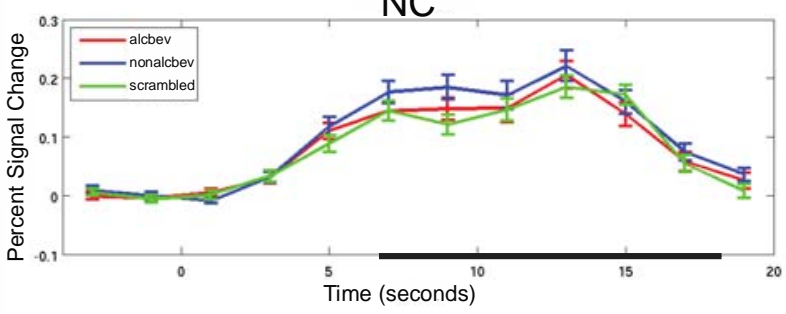

D. Left Fusiform

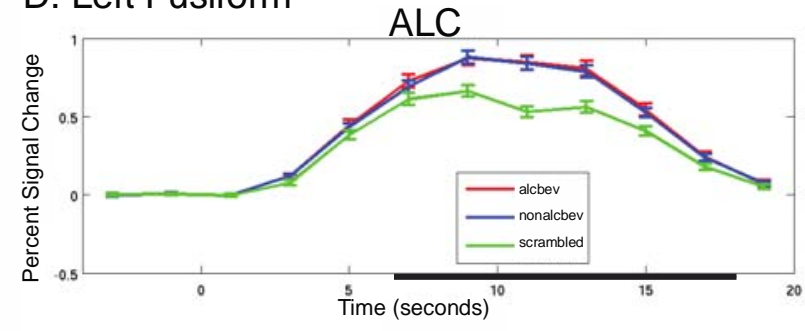

NC

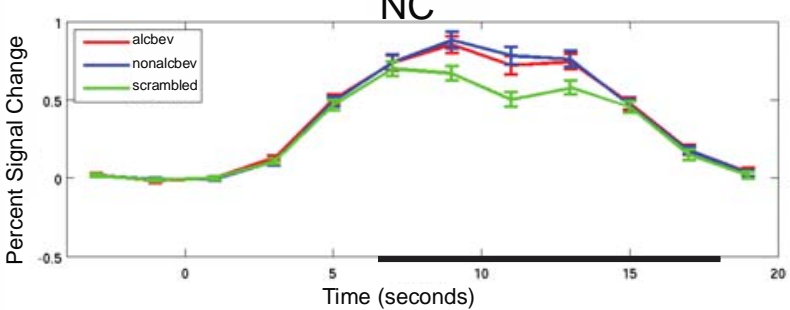

\section{2 by distractor type}

833 The percent signal change represents brain activity during presentation of fixation and the task stimuli. Error bars

834 represent the standard error of the mean. Time zero was set to the onset of the encoded faces, and signal zero was set

835 to the average signal for the three initial time points (two pre-trial and one post-trial onset). The analysis window

836 used to examine the distractor was 6 to 16 seconds following trial onset (2 to 12 seconds after distractor onset), as

837 indicated by the thick line on the $\mathrm{x}$ axis. Abbreviations: $\mathrm{OFC}=$ orbitofrontal cortex; VLPFC $=$ ventrolateral

838 prefrontal cortex; $\mathrm{ACC}=$ anterior cingulate cortex. The ACC (Panel C) is part of the anterior hub within the

839 fixation-regions. The remaining areas are primarily task-regions. 


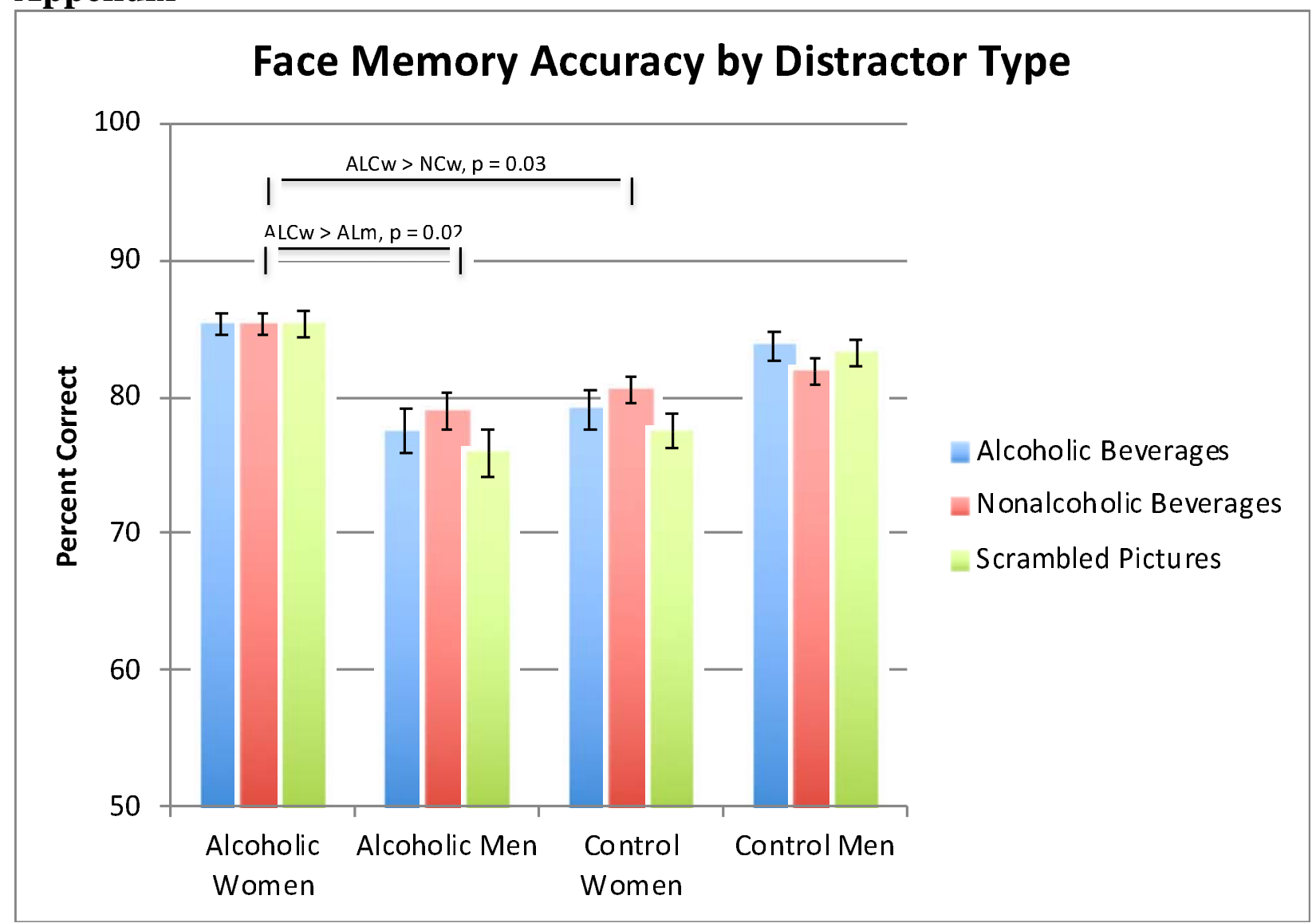

842 Figure A1. Group and gender comparisons in percent correct responses to the probe face

843 A significant Group-by-Gender interaction showed that face memory accuracy was significantly higher for the 844 Alcoholic Women than the Alcoholic Men, a gender difference that was greater than the one observed for the NC group. Alcoholic Women also had higher accuracy than Control Women. (Also see Table A1.) There was no significant effect of Distractor type on performance accuracy. Error bars represent standard error of the mean. 


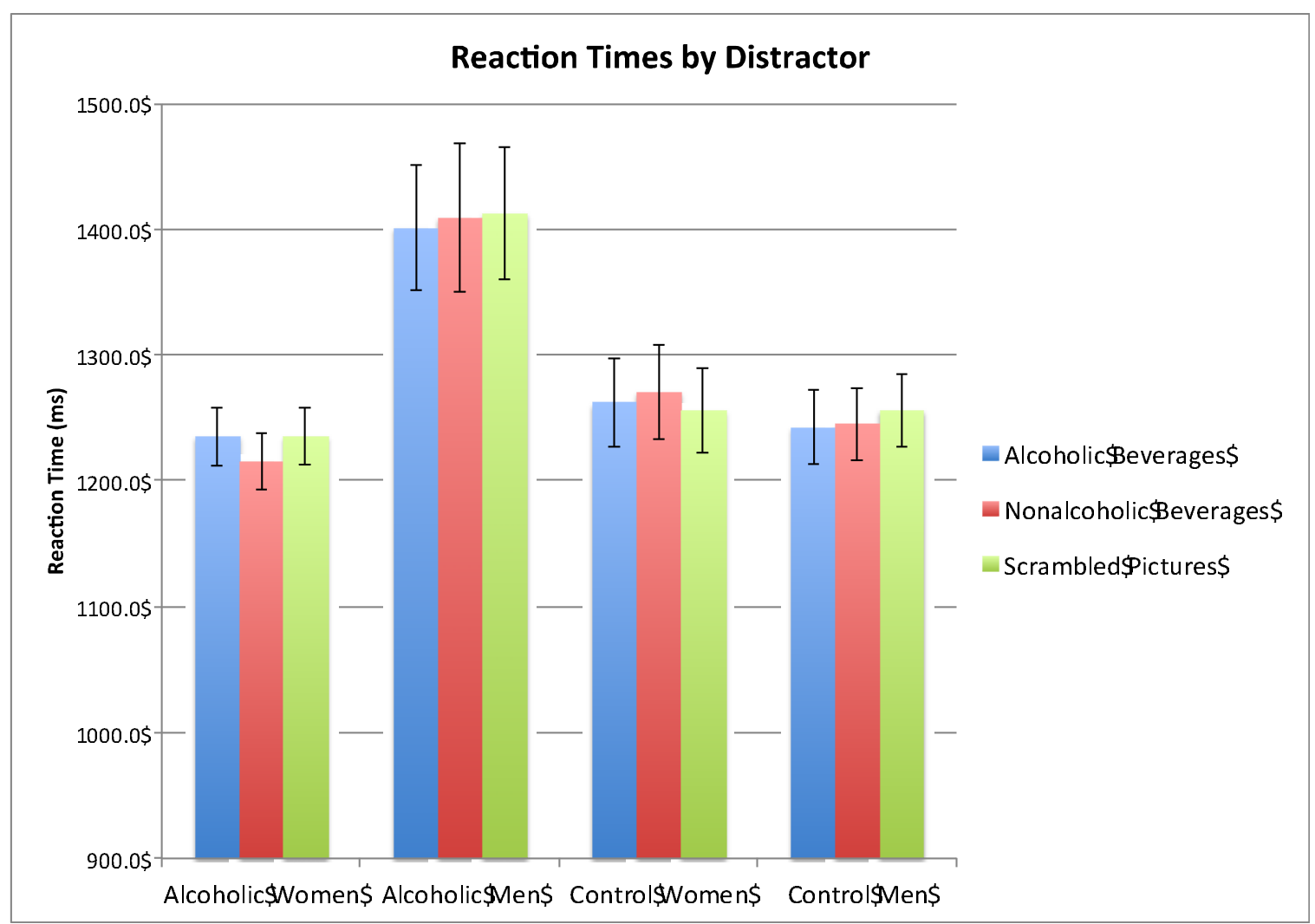

850 Figure A2. Face memory reaction times: group and gender comparisons in reaction times to the probe face as a function of the distractor stimuli

852 Reaction times (in milliseconds) are shown for correct trials sorted by conditions and groups. (Also see Table A2.) Participants did not vary significantly by Group or Gender on overall reaction times. There was no significant effect of Distractor type on reaction time, nor did reaction time performance by Distractor type significantly vary by Group 

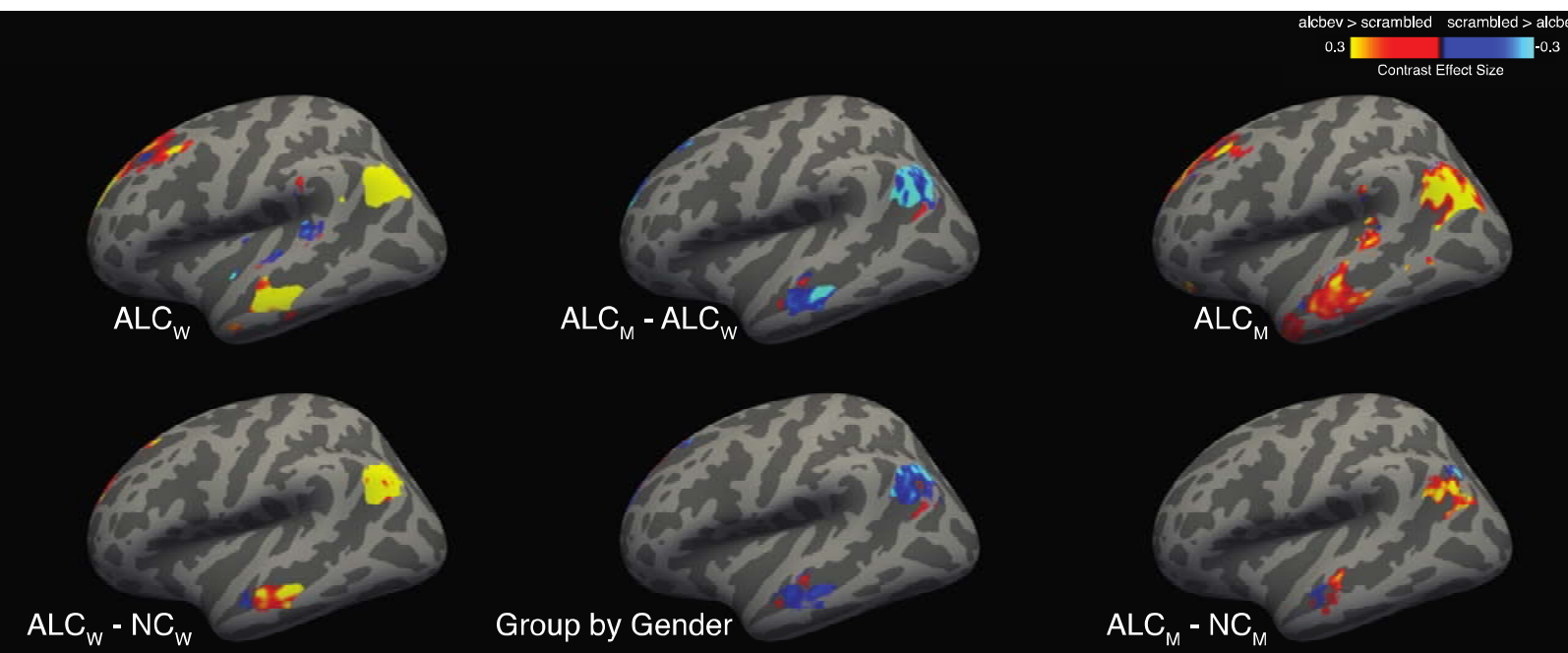

Group by Gender

$$
\mathrm{ALC}_{\mathrm{M}}-\mathrm{NC}_{\mathrm{M}}
$$

\section{Figure A3. Cortical activati
regions (left lateral view)}

A significant Group-by-Gender interaction revealed several clusters (see Figure 3 and Table A4), although no clusters with significant interactions were found on the left lateral surface. Group mean contrast values (for alcbev vs. scrambled within fixation-regions) are displayed in the four brain images located in the corners of the figure, and group comparisons are indicated by minus signs. Abbreviations: $\mathrm{ALCm}=$ Alcoholic men; $\mathrm{ALCw}=\mathrm{Alcoholic}$ 

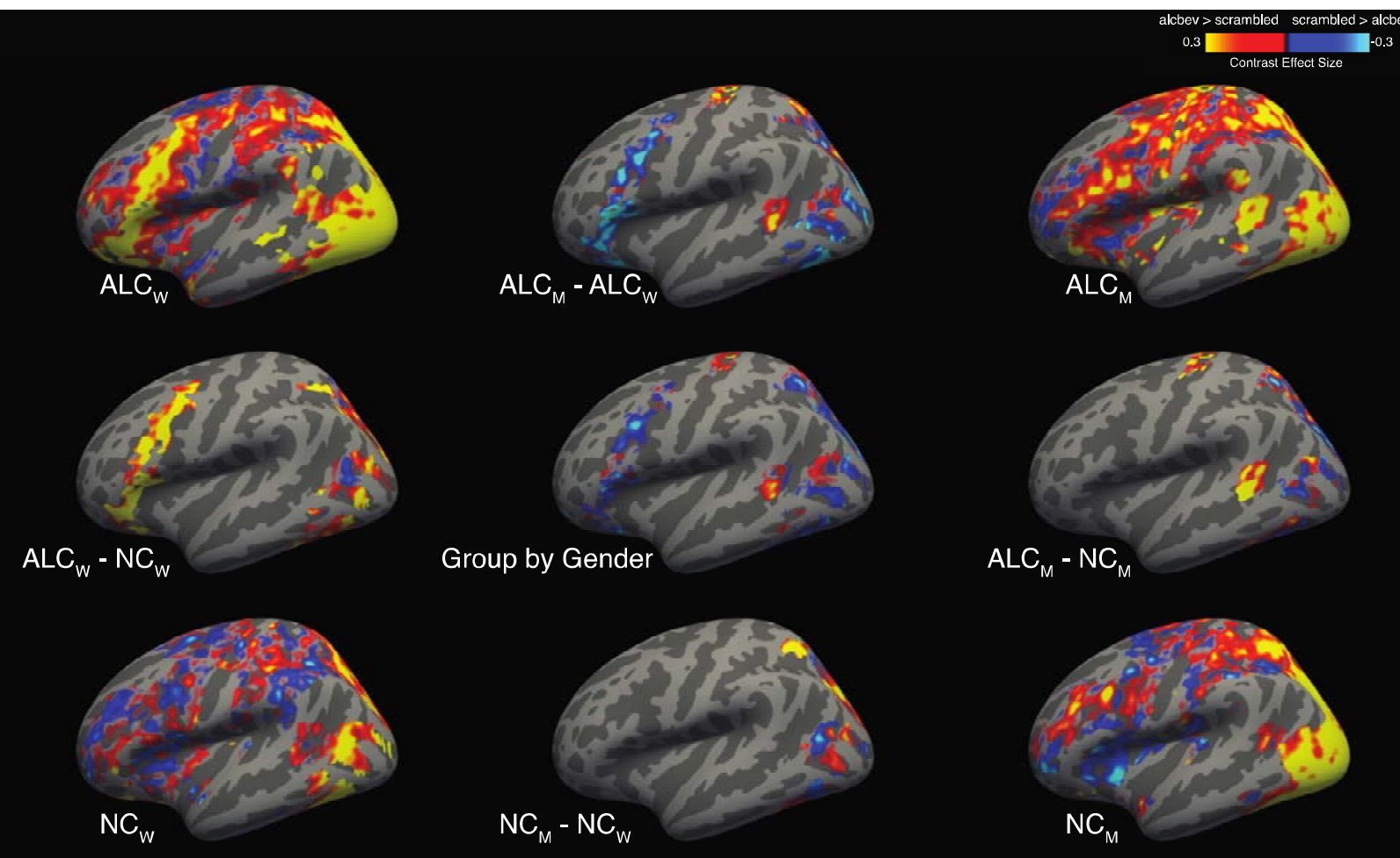

$\mathrm{ALC}_{\mathrm{M}}-\mathrm{NC}_{\mathrm{M}}$

\section{Figure A4. Cortical activation maps for the alcbev vs. scrambled contrast within task- regions (left lateral view)}

A significant Group-by-Gender interaction revealed several clusters (see Figure 4 and Table A4), although none are visible on the left lateral view. Group mean contrast values (for alcbev vs. scrambled within fixation-regions) are displayed in the four brain images located in the corners of the figure, and group comparisons are indicated by minus signs. Abbreviations: $\mathrm{ALCm}=$ Alcoholic men; $\mathrm{ALCw}=$ Alcoholic women; $\mathrm{NCm}=$ Nonalcoholic men; $\mathrm{NCw}$ $=$ Nonalcoholic women. 


\begin{tabular}{|c|c|c|c|c|c|c|}
\hline \multicolumn{7}{|c|}{ Percent Correct Responses Overall ${ }^{\text {abc }}$} \\
\hline & ALC & ALCw & ALCm & $\mathrm{NC}$ & $\mathrm{NCw}$ & $\mathrm{NCm}$ \\
\hline Mean & 81.4 & 85.3 & 77.4 & 81.0 & 79.0 & 82.9 \\
\hline Standard Deviation & 11.5 & 6.8 & 13.8 & 9.7 & 10.7 & 8.4 \\
\hline Range & $50.0-97.5$ & $66.0-96.3$ & $50.0-97.5$ & $54.9-96.9$ & $54.9-92.6$ & $71.0-96.9$ \\
\hline \multicolumn{7}{|c|}{ Percent Correct Responses by Distractor } \\
\hline & ALC & $\mathrm{ALCW}$ & ALCm & $\mathrm{NC}$ & $\mathrm{NCw}$ & $\mathrm{NCm}$ \\
\hline \multicolumn{7}{|l|}{ Alcoholic Beverages } \\
\hline Mean & 81.4 & 85.3 & 77.5 & 81.4 & 79.1 & 83.7 \\
\hline Standard Deviation & 12.4 & 7.4 & 15.0 & 11.4 & 12.9 & 9.5 \\
\hline Range & $45.8-98.1$ & $66.7-96.3$ & $45.8-98.1$ & $51.9-98.1$ & $51.9-98.1$ & $64.8-98.1$ \\
\hline \multicolumn{7}{|l|}{ Nonalcoholic Beverages } \\
\hline Mean & 82.1 & 85.3 & 78.9 & 81.1 & 80.5 & 81.8 \\
\hline Standard Deviation & 10.8 & 7.7 & 12.5 & 9.0 & 9.2 & 9.0 \\
\hline Range & $53.7-98.1$ & $68.8-98.1$ & $53.7-96.3$ & $57.4-94.4$ & $57.4-91.7$ & $64.8-94.4$ \\
\hline \multicolumn{7}{|l|}{ Scrambled Pictures } \\
\hline Mean & 80.6 & 85.3 & 75.9 & 80.4 & 77.5 & 83.2 \\
\hline Standard Deviation & 13.6 & 8.6 & 16.1 & 11.1 & 12.1 & 9.4 \\
\hline Range & $44.4-98.1$ & $62.5-98.1$ & $44.4-98.1$ & $51.9-98.1$ & $51.9-91.7$ & $61.1-98.1$ \\
\hline \multicolumn{7}{|c|}{ Percent Correct Responses by Facial Emotion $^{\text {def }}$} \\
\hline & ALC & ALCw & ALCm & $\mathrm{NC}$ & $\mathrm{NCw}$ & $\mathrm{NCm}$ \\
\hline \multicolumn{7}{|l|}{ Positive Faces } \\
\hline Mean & 82.4 & 86.8 & 78.1 & 80.7 & 79.4 & 82.0 \\
\hline Standard Deviation & 12.5 & 9.0 & 14.2 & 10.4 & 11.9 & 8.7 \\
\hline Range & $51.9-100.0$ & $56.3-100.0$ & $51.9-96.3$ & $51.9-96.3$ & $51.9-94.4$ & $70.4-96.3$ \\
\hline \multicolumn{7}{|l|}{ Negative Faces } \\
\hline Mean & 81.9 & 85.9 & 77.8 & 82.9 & 80.9 & 84.9 \\
\hline Standard Deviation & 12.0 & 7.2 & 14.4 & 9.5 & 11.0 & 7.5 \\
\hline Range & $46.3-98.1$ & $68.8-98.1$ & $46.3-98.1$ & $53.7-98.1$ & $53.7-94.4$ & $72.2-98.1$ \\
\hline \multicolumn{7}{|l|}{ Neutral Faces } \\
\hline Mean & 79.8 & 83.2 & 76.4 & 79.3 & 76.7 & 81.9 \\
\hline Standard Deviation & 11.8 & 7.5 & 14.3 & 11.2 & 11.1 & 10.9 \\
\hline Range & $51.9-98.1$ & $62.5-92.6$ & $51.9-98.1$ & $50.0-96.3$ & $50.0-92.6$ & $63.0-96.3$ \\
\hline
\end{tabular}

\section{Table A1. Behavioral task percent correct responses}

878 Scores for accuracy are provided for overall performance, distractor type, and facial emotion. ${ }^{\mathrm{a}}$ Group x Gender;

879 Alcoholic Women > Alcoholic Men; ${ }^{\mathrm{c}}$ Alcoholic Women > Control Women; ${ }^{\mathrm{d}}$ Emotion main effect; ${ }^{\mathrm{e}}$ Positive Faces

$880>$ Neutral Faces; ${ }^{\mathrm{f}}$ Negative Faces $>$ Neutral Faces; all $p<0.5$. Abbreviations: ALCw $=$ Alcoholic Women; ALCm $=$

881 Alcoholic Men; $\mathrm{NCw}=$ Nonalcoholic Control Women; $\mathrm{NCm}=$ Nonalcoholic Control Men.

882 


\begin{tabular}{|c|c|c|c|c|c|c|}
\hline & $\begin{array}{l}\text { ALC } \\
n=42\end{array}$ & $\begin{array}{l}\mathrm{NC} \\
\mathrm{n}=42\end{array}$ & $\begin{array}{l}\text { ALCw } \\
n=21\end{array}$ & $\begin{array}{l}\text { ALCm } \\
\mathrm{n}=21\end{array}$ & $\begin{array}{l}\mathrm{NCw} \\
\mathrm{n}=21\end{array}$ & $\begin{array}{l}\mathrm{NCm} \\
\mathrm{n}=21\end{array}$ \\
\hline \multicolumn{7}{|l|}{ All Trials (ms) } \\
\hline mean & 1374.0 & 1302.9 & 1263.4 & 1484.6 & 1319.3 & 1286.5 \\
\hline standard deviation & 418.2 & 300.0 & 213.0 & 536.1 & 337.2 & 265.0 \\
\hline range & $896.6-2720.8$ & $799.6-2164.9$ & $896.6-1699.5$ & $922.8-2720.8$ & $931.2-2164.9$ & $799.6-1812.7$ \\
\hline \multicolumn{7}{|l|}{ Correct Trials (ms) } \\
\hline mean & 1317.6 & 1254.9 & 1229.4 & 1405.9 & 1263.1 & 1246.7 \\
\hline standard deviation & 377.0 & 288.1 & 207.9 & 481.5 & 321.1 & 258.7 \\
\hline range & $889.7-2454.1$ & $784.2-2129.2$ & $889.7-1649.3$ & $912.4-2454.1$ & $909.0-2129.2$ & $784.2-1795.1$ \\
\hline \multicolumn{7}{|l|}{ Incorrect Trials (ms) } \\
\hline mean & 1686.2 & 1564.2 & 1532.4 & 1840.0 & 1591.0 & 1537.5 \\
\hline standard deviation & 538.2 & 328.2 & 285.2 & 680.3 & 376.8 & 278.1 \\
\hline range & $1079.8-3883.9$ & $1064.2-2480.1$ & $1160.8-2374.8$ & $1079.8-3883.9$ & $1064.2-2480.1$ & $1097.1-1939.1$ \\
\hline & ALC & $\mathrm{NC}$ & $\mathrm{ALCw}$ & $\mathrm{ALCm}$ & $\mathrm{NCw}$ & $\mathrm{NCm}$ \\
\hline \multicolumn{7}{|l|}{ Alcoholic Beverages (ms) } \\
\hline mean & 1318.3 & 1252.2 & 1235.4 & 1401.1 & 1261.7 & 1242.7 \\
\hline standard deviation & 360.9 & 292.1 & 216.4 & 453.6 & 320.2 & 268.6 \\
\hline range & $866.2-2367.9$ & $765.7-2101.9$ & $866.2-1578.8$ & $926.1-2367.9$ & $906.8-2101.9$ & $765.7-1837.5$ \\
\hline \multicolumn{7}{|l|}{ Nonalcoholic Beverages (ms) } \\
\hline mean & 1312.4 & 1257.5 & 1215.5 & 1409.3 & 1270.6 & 1244.5 \\
\hline standard deviation & 419.1 & 304.9 & 208.2 & 545.0 & 346.9 & 264.4 \\
\hline range & $886.8-2682.0$ & $779.7-2274.0$ & $886.8-1686.6$ & $902.8-2682.0$ & $910.8-2274.0$ & $779.7-1894.5$ \\
\hline \multicolumn{7}{|l|}{ Scrambled Pictures (ms) } \\
\hline mean & 1324.2 & 1255.9 & 1235.7 & 1412.7 & 1255.8 & 1256.0 \\
\hline standard deviation & 378.8 & 281.8 & 213.1 & 482.0 & 306.5 & 262.5 \\
\hline range & $888.7-2678.9$ & 806.6 - 2004.1 & $914.9-1686.1$ & $888.7-2678.9$ & $882.4-2004.1$ & $806.6-1825.8$ \\
\hline & ALC & $\mathrm{NC}$ & $\mathrm{ALCW}$ & ALCm & $\mathrm{NCw}$ & $\mathrm{NCm}$ \\
\hline \multicolumn{7}{|l|}{ Positive Faces (ms) } \\
\hline mean & 1290.5 & 1241.2 & 1227.9 & 1353.1 & 1238.4 & 1244.0 \\
\hline standard deviation & 347.2 & 279.5 & 229.3 & 431.6 & 306.7 & 257.0 \\
\hline range & $867.7-2608.6$ & $789.2-2015.1$ & $867.7-1617.1$ & $894.3-2608.6$ & $870.0-2015.1$ & $789.2-1871.9$ \\
\hline \multicolumn{7}{|l|}{ Negative Faces (ms) } \\
\hline mean & 1303.9 & 1251.2 & 1225.7 & 1382.0 & 1264.3 & 1238.0 \\
\hline standard deviation & 322.5 & 294.0 & 186.2 & 407.2 & 323.2 & 269.1 \\
\hline range & $900.0-2218.6$ & $778.9-2128.5$ & $900.0-1674.6$ & $918.8-2218.6$ & $889.4-2128.5$ & $778.9-1803.4$ \\
\hline \multicolumn{7}{|l|}{ Neutral Faces (ms) } \\
\hline mean & 1356.4 & 1272.4 & 1233.6 & 1479.2 & 1286.8 & 1258.0 \\
\hline standard deviation & 492.4 & 307.0 & 235.3 & 640.3 & 346.8 & 269.4 \\
\hline range & $857.7-3051.0$ & $784.4-2226.0$ & $857.7-1724.7$ & $887.4-3051.0$ & $875.3-2226.0$ & $784.4-1706.6$ \\
\hline & ALC & $\mathrm{NC}$ & $\mathrm{ALCw}$ & $\mathrm{ALCm}$ & $\mathrm{NCw}$ & $\mathrm{NCm}$ \\
\hline \multicolumn{7}{|l|}{ Female Faces (ms) } \\
\hline mean & 1321.8 & 1272.3 & 1237.9 & 1405.7 & 1275.2 & 1269.4 \\
\hline standard deviation & 369.4 & 288.7 & 206.9 & 471.3 & 311.5 & 271.8 \\
\hline range & $903.8-2648.9$ & $812.7-2089.0$ & $903.8-1692.7$ & $930.6-2648.9$ & $919.7-2089.0$ & $812.7-1830.5$ \\
\hline \multicolumn{7}{|l|}{ Male Faces (ms) } \\
\hline mean & 1314.0 & 1237.0 & 1220.4 & 1407.6 & 1249.7 & 1224.3 \\
\hline standard deviation & 403.4 & 292.6 & 212.4 & 519.7 & 332.7 & 254.0 \\
\hline range & $872.0-2797.2$ & $756.1-2159.7$ & $875.7-1605.9$ & $872.0-2797.2$ & $880.1-2159.7$ & $756.1-1826.1$ \\
\hline
\end{tabular}

\section{Table A2. Reaction times}

885 Group reaction times in milliseconds are provided for distractor type, facial emotion, and face gender. Footnotes

886 indicate significant differences, all $p<0.5$ : ${ }^{\mathrm{a}}$ Group x Gender interaction; ${ }^{\mathrm{b}}$ Alcoholic Women $>$ Alcoholic Men;

887 'Alcoholic Women > Control Women; ${ }^{\mathrm{d}}$ Emotion main effect; ${ }^{\mathrm{e}}$ Positive Faces > Neutral Faces; ${ }^{\mathrm{f}}$ Negative Faces >

888 Neutral Faces. Abbreviations: $\mathrm{ALCw}=$ Alcoholic Women; $\mathrm{ALCm}=$ Alcoholic Men; $\mathrm{NCw}=$ Nonalcoholic Control

889 Women; $\mathrm{NCm}=$ Nonalcoholic Control Men. 


\begin{tabular}{|c|c|c|c|c|c|c|c|}
\hline Group & Fixation Masking & Contrast & Annotation & HemiMax & VtxMax & Size $\left(\mathrm{mm}^{\wedge} 2\right)$ & MNIX \\
\hline$\overline{\mathrm{ALCW}}$ & fixation-regions & alcbev > nonalcbev & inferiorparietal & 5.777 & 78109 & 894.36 & -46.9 \\
\hline $\mathrm{ALCW}$ & fixation-regions & alcbev > nonalcbev & superiorfrontal & 5.198 & 117174 & 2021.3 & -7.3 \\
\hline $\mathrm{ALCw}$ & fixation-regions & alcbev > nonalcbev & precuneus & 4.279 & 104685 & 1114.58 & -6.4 \\
\hline $\mathrm{ALCW}$ & fixation-regions & alcbev $>$ nonalcbev & middletemporal & 3.397 & 132409 & 372.86 & -57.9 \\
\hline $\mathrm{ALCW}$ & fixation-regions & alcbev > nonalcbev & precuneus & 5.031 & 78184 & 343.04 & 8.9 \\
\hline $\mathrm{ALCW}$ & fixation-regions & alcbev > nonalcbev & superiorfrontal & 3.007 & 33332 & 470.55 & 7.4 \\
\hline $\mathrm{ALCW}$ & fixation-regions & alcbev $>$ nonalcbev & middletemporal & 2.388 & 149193 & 390.07 & 57.3 \\
\hline $\mathrm{NCm}$ & fixation-regions & alcbev > nonalcbev & precuneus & 2.001 & 28044 & 372.78 & -5.8 \\
\hline $\mathrm{NCm}$ & fixation-regions & alcbev > nonalcbev & inferiorparietal & 2.093 & 87534 & 353.25 & 48 \\
\hline $\mathrm{NCw}$ & fixation-regions & alcbev > nonalcbev & rostralanteriorcingulate & 3.853 & 72938 & 1014.62 & -5.7 \\
\hline $\mathrm{NCw}$ & fixation-regions & alcbev > nonalcbev & precuneus & 3.518 & 88451 & 552.66 & -6.9 \\
\hline $\mathrm{NCw}$ & fixation-regions & alcbev > nonalcbev & superiorfrontal & 3.043 & 106164 & 573.38 & -7.2 \\
\hline $\mathrm{NCw}$ & fixation-regions & alcbev > nonalcbev & superiorfrontal & 2.719 & 37125 & 231.3 & -13.6 \\
\hline $\mathrm{NCw}$ & fixation-regions & alcbev > nonalcbev & superiorfrontal & 3.812 & 154767 & 425.34 & 11.2 \\
\hline $\mathrm{NCw}$ & fixation-regions & alcbev > nonalcbev & superiorfrontal & 2.997 & 141183 & 288 & 9.1 \\
\hline $\mathrm{NCw}$ & fixation-regions & alcbev > nonalcbev & precuneus & 2.381 & 20851 & 168.89 & 12.5 \\
\hline $\mathrm{ALCm}$ & fixation-regions & alcbev > scrambled & superiortemporal & 3.628 & 127514 & 287.34 & -52.7 \\
\hline $\mathrm{ALCm}$ & fixation-regions & alcbev > scrambled & inferiorparietal & 3.532 & 77987 & 597.66 & -39.4 \\
\hline $\mathrm{ALCm}$ & fixation-regions & alcbev > scrambled & precuneus & 3.011 & 42213 & 1099.78 & -4.6 \\
\hline $\mathrm{ALCm}$ & fixation-regions & alcbev > scrambled & temporalpole & 3.202 & 75073 & 255.04 & 36.1 \\
\hline $\mathrm{ALCm}$ & fixation-regions & alcbev > scrambled & middletemporal & 2.184 & 32116 & 246.83 & 55.8 \\
\hline $\mathrm{ALCW}$ & fixation-regions & alcbev > scrambled & superiorfrontal & 6.192 & 119696 & 1479.43 & -9 \\
\hline $\mathrm{ALCW}$ & fixation-regions & alcbev > scrambled & isthmuscingulate & 4.52 & 21771 & 1599.1 & -4.6 \\
\hline $\mathrm{ALCW}$ & fixation-regions & alcbev > scrambled & inferiorparietal & 3.772 & 8342 & 1007.44 & -46.3 \\
\hline $\mathrm{ALCW}$ & fixation-regions & alcbev > scrambled & medialorbitofrontal & 3.625 & 141106 & 792.29 & -10.2 \\
\hline $\mathrm{ALCW}$ & fixation-regions & alcbev $>$ scrambled & middletemporal & 3.4 & 132407 & 461.02 & -57.4 \\
\hline $\mathrm{ALCW}$ & fixation-regions & alcbev > scrambled & precuneus & 4.528 & 147039 & 407.44 & 6 \\
\hline $\mathrm{ALCw}$ & fixation-regions & alcbev $>$ scrambled & rostralmiddlefrontal & 2.928 & 77385 & 752.89 & 18.2 \\
\hline $\mathrm{ALCW}$ & fixation-regions & alcbev $>$ scrambled & superiorfrontal & 2.644 & 116693 & 213.6 & 7.6 \\
\hline $\mathrm{NCm}$ & fixation-regions & alcbev > scrambled & isthmuscingulate & 4.044 & 159377 & 1127.18 & -7.7 \\
\hline $\mathrm{NCm}$ & fixation-regions & alcbev $>$ scrambled & middletemporal & 3.081 & 46308 & 253.13 & -57.3 \\
\hline $\mathrm{NCm}$ & fixation-regions & alcbev $>$ scrambled & medialorbitofrontal & 2.523 & 119804 & 386.08 & -7.7 \\
\hline $\mathrm{NCm}$ & fixation-regions & alcbev > scrambled & superiorfrontal & 2.157 & 34872 & 247.83 & -9.7 \\
\hline $\mathrm{NCm}$ & fixation-regions & alcbev > scrambled & inferiorparietal & 2.069 & 117757 & 286.31 & -34.4 \\
\hline $\mathrm{NCm}$ & fixation-regions & alcbev $>$ scrambled & inferiorparietal & 3.908 & 130241 & 606.24 & 46.4 \\
\hline $\mathrm{NCm}$ & fixation-regions & alcbev > scrambled & superiorfrontal & 3.772 & 154660 & 398.31 & 9.2 \\
\hline $\mathrm{NCm}$ & fixation-regions & alcbev $>$ scrambled & isthmuscingulate & 2.376 & 85932 & 344.91 & 6.8 \\
\hline $\mathrm{NCm}$ & fixation-regions & alcbev > scrambled & superiorfrontal & 2.322 & 18118 & 261.88 & 16.4 \\
\hline $\mathrm{NCw}$ & fixation-regions & alcbev $>$ scrambled & superiorfrontal & 3.775 & 145688 & 785.15 & -9.1 \\
\hline $\mathrm{NCw}$ & fixation-regions & alcbev $>$ scrambled & medialorbitofrontal & 3.35 & 98461 & 869.42 & -9.6 \\
\hline $\mathrm{NCw}$ & fixation-regions & alcbev > scrambled & middletemporal & 2.379 & 13182 & 203.24 & -51.4 \\
\hline $\mathrm{ALCm}$ & fixation-regions & nonalcbev > scrambled & superiortemporal & 3.138 & 161045 & 299.84 & -48.8 \\
\hline $\mathrm{ALCw}$ & fixation-regions & nonalcbev > scrambled & rostralmiddlefrontal & 3.585 & 82490 & 424.57 & -18.9 \\
\hline $\mathrm{ALCw}$ & fixation-regions & nonalcbev > scrambled & superiorfrontal & 2.628 & 35019 & 244.79 & 14.4 \\
\hline $\mathrm{NCm}$ & fixation-regions & nonalcbev > scrambled & superiorfrontal & 3.123 & 149679 & 531.99 & 10.9 \\
\hline $\mathrm{NCm}$ & fixation-regions & nonalcbev > scrambled & caudalmiddlefrontal & 2.592 & 63876 & 228.53 & 32 \\
\hline $\mathrm{ALCm}$ & task-regions & alcbev > nonalcbev & precuneus & 3.84 & 30022 & 820.45 & -9.8 \\
\hline $\mathrm{ALCm}$ & task-regions & alcbev > nonalcbev & inferiorparietal & 3.068 & 156958 & 724.95 & -46.8 \\
\hline $\mathrm{ALCm}$ & task-regions & alcbev > nonalcbev & lateraloccipital & 2.796 & 46455 & 556.39 & 41.8 \\
\hline $\mathrm{ALCW}$ & task-regions & alcbev $>$ nonalcbev & fusiform & 4.553 & 127987 & 3039.55 & -41.4 \\
\hline $\mathrm{ALCw}$ & task-regions & alcbev > nonalcbev & lateralorbitofrontal & 3.917 & 9114 & 696 & -32.3 \\
\hline $\mathrm{ALCW}$ & task-regions & alcbev > nonalcbev & parsopercularis & 3.709 & 110686 & 543.82 & -46.6 \\
\hline $\mathrm{ALCw}$ & task-regions & alcbev > nonalcbev & inferiorparietal & 3.231 & 29327 & 517.43 & -44.1 \\
\hline $\mathrm{ALCw}$ & task-regions & alcbev > nonalcbev & inferiorparietal & 2.89 & 158246 & 972.39 & 42.5 \\
\hline $\mathrm{NCw}$ & task-regions & alcbev > nonalcbev & superiorparietal & 2.749 & 100056 & 696.49 & -21.3 \\
\hline $\mathrm{ALCm}$ & task-regions & alcbev > scrambled & lateraloccipital & 6.82 & 112623 & 9254.88 & -23.5 \\
\hline $\mathrm{ALCm}$ & task-regions & alcbev $>$ scrambled & precentral & 5.097 & 102385 & 1154.47 & -27.7 \\
\hline $\mathrm{ALCm}$ & task-regions & alcbev > scrambled & bankssts & 3.981 & 86601 & 489.82 & -50.3 \\
\hline $\mathrm{ALCm}$ & task-regions & alcbev > scrambled & lateraloccipital & 5.32 & 129176 & 5250.06 & 25.7 \\
\hline $\mathrm{ALCm}$ & task-regions & alcbev $>$ scrambled & precentral & 2.656 & 72522 & 782.86 & 42.6 \\
\hline $\mathrm{ALCw}$ & task-regions & alcbev > scrambled & fusiform & 6.095 & 34311 & 13105.37 & -29.1 \\
\hline $\mathrm{ALCW}$ & task-regions & alcbev > scrambled & isthmuscingulate & 4.555 & 57611 & 563.79 & -7.7 \\
\hline $\mathrm{ALCW}$ & task-regions & alcbev > scrambled & parsopercularis & 4.416 & 20526 & 2644.35 & -49.9 \\
\hline $\mathrm{ALCw}$ & task-regions & lcbev > scrambled & superiorfrontal & 3.915 & 133846 & 522.69 & -6.6 \\
\hline $\mathrm{ALCW}$ & task-regions & alcbev $>$ scrambled & lateraloccipital & 8.832 & 119142 & 12539.17 & 28.1 \\
\hline $\mathrm{ALCW}$ & task-regions & alcbev > scrambled & superiorfrontal & 3.963 & 47146 & 785.28 & 7.9 \\
\hline $\mathrm{ALCw}$ & task-regions & lcbev > scrambled & lateralorbitofrontal & 3.159 & 37696 & 1035.15 & 39.4 \\
\hline $\mathrm{ALCW}$ & task-regions & alcbev $>$ scrambled & rostralmiddlefrontal & 2.655 & 37107 & 1604.06 & 40 \\
\hline $\mathrm{NCm}$ & task-regions & alcbev > scrambled & lateraloccipital & 6.805 & 122808 & 11030.59 & -15.2 \\
\hline $\mathrm{NCm}$ & task-regions & lcbev > scrambled & fusiform & 6.25 & 92502 & 10153.11 & 35.9 \\
\hline $\mathrm{NCm}$ & task-regions & alcbev > scrambled & precuneus & 2.429 & 98061 & 571.75 & 10.4 \\
\hline $\mathrm{NCw}$ & task-regions & cbev > scrambled & fusiform & 4.853 & 48274 & 2961.14 & -29.9 \\
\hline $\mathrm{NCw}$ & task-regions & alcbev $>$ scrambled & inferiornarietal & 3.525 & 45896 & 469.12 & -43.9 \\
\hline $\mathrm{NCw}$ & task-regions & alcbev > scrambled & superiorparietal & 2.819 & 157385 & 433.04 & -24.2 \\
\hline $\mathrm{NCw}$ & task-regions & alcbev > scrambled & superiorparietal & 2.682 & 87111 & 609.56 & -25.6 \\
\hline $\mathrm{NCw}$ & task-regions & alcbev > scrambled & lateraloccipital & 6.04 & 90488 & 1846.73 & 25.4 \\
\hline $\mathrm{NCw}$ & task-regions & alcbev > scrambled & lateraloccipital & 3.065 & 114637 & 624.55 & 44.2 \\
\hline $\mathrm{NCw}$ & task-regions & alcbev > scrambled & superiorparietal & 2.97 & 157814 & 450.45 & 31.1 \\
\hline $\mathrm{ALCm}$ & task-regions & nonalcbev > alcbev & nericalcarine & -3.202 & 120908 & 591.21 & -9.1 \\
\hline $\mathrm{ALCm}$ & task-regions & nonalcbev > alcbev & pericalcarine & -1.915 & 43525 & 692.03 & 17.9 \\
\hline $\mathrm{NCm}$ & task-regions & nonalcbev > alcbev & lateraloccipital & -2.977 & 38531 & 778.52 & -37.6 \\
\hline $\mathrm{NCm}$ & task-regions & nonalcbev > alcbev & incula & -2.786 & 96569 & 492.26 & -32.9 \\
\hline $\mathrm{NCm}$ & task-regions & nonalcbev > alcbev & superiorfrontal & -2.596 & 67709 & 754.6 & -9.5 \\
\hline $\mathrm{ALCm}$ & task-regions & nonalcbev > scrambled & lateraloccipital & 6.96 & 148912 & 5460.13 & -22.1 \\
\hline $\mathrm{ALCm}$ & task-regions & nonalcbev > scrambled & lateraloccipital & 5.663 & 62800 & 4195.98 & 20.1 \\
\hline $\mathrm{ALCm}$ & task-regions & nonalcbev > scrambled & superiorfrontal & 3.639 & 70680 & 521.47 & 17.4 \\
\hline $\mathrm{ALCw}$ & task-regions & nonalcbev > scrambled & lateraloccipital & 6.88 & 8571 & 8685.5 & -21.4 \\
\hline $\mathrm{ALCW}$ & task-regions & nonalcbev > scrambled & fusiform & 9.645 & 107788 & 9693.99 & 33.9 \\
\hline $\mathrm{NCm}$ & task-regions & nonalcbev > scrambled & lateraloccipital & 9.652 & 49589 & 11945.19 & -12 \\
\hline $\mathrm{NCm}$ & task-regions & nonalcbev > scrambled & lateraloccipital & 7.109 & 71808 & 9794.62 & 28.8 \\
\hline
\end{tabular}




\section{Table A3. Cortical cluster characteristics for significant contrasts within each group}

894 Annotations (from the peak voxel location in the Desikan-Killiany atlas) are shown for each of the 99 clusters with 895 significant distractor contrasts, calculated for each group separately. The clusters reported can be understood to span 896 multiple functional regions (Woo et al., 2014). That is, they are not limited to a single region, as reported by the 897 maximal vertex or voxel. Abbreviations: $\mathrm{Hemi}=$ hemisphere; $\operatorname{Max}=\operatorname{maximum}-\log _{10}(p$-value $)$ for group comparison in the cluster; VtxMax = vertex number at the maximum; Size = surface area of cluster; MNIX, MNIY, and MNIZ = Montreal Neurological Institute 305-subject template coordinates X, Y, and Z for the maximum vertex; CWP $=$ cluster-wise $p$-value further corrected for the three spaces of left cortex, right cortex, and volume; CWPLow and CWPHi $=90 \%$ confidence intervals for CWP; NVtxs = number of vertices in the cluster; alcbev $=$ alcoholic

903 beverages; nonalcbev $=$ nonalcoholic beverages; $\mathrm{L}=$ left hemisphere; $\mathrm{R}=$ right hemisphere; bankssts $=$ banks of the 


\begin{tabular}{|c|c|c|c|c|c|c|c|c|c|c|c|c|c|}
\hline Fixation Masking & Contrast & Annotation & Hemi & $\operatorname{Max}$ & VtxMax & Size $\left(\mathrm{mm}^{\wedge} 2\right)$ & MNIX & MNIY & MNIZ & CWP & CWPLow & CWPHi & NVtxs \\
\hline fixation-regions & alcbev > scrambled & superiorfrontal & $\mathrm{L}$ & -2.635 & 55177 & 313.89 & -7.9 & 46.2 & 22.9 & 0.01136 & 0.00897 & 0.01374 & 461 \\
\hline fixation-regions & alcbev > scrambled & superiorfrontal & $\mathrm{R}$ & -3.304 & 146084 & 140.84 & 16.5 & 53.5 & 24.4 & 0.0021 & 0.0012 & 0.003 & 201 \\
\hline fixation-regions & alcbev > scrambled & superiorfrontal & $\mathrm{R}$ & -2.453 & 134468 & 116.29 & 12 & 47.4 & 13.5 & 0.01017 & 0.00808 & 0.01255 & 184 \\
\hline fixation-regions & alcbev $>$ scrambled & ecuneus & $\mathrm{R}$ & -1.444 & 122115 & 162.46 & 9.6 & -54.5 & 24.9 & 0.0003 & 0 & 0.0006 & 418 \\
\hline fixation-regions & nonalcbev > scrambled & periorfrontal & $\mathrm{R}$ & 3.422 & 59361 & 133.19 & 7.6 & 44.3 & 38.6 & 0.0003 & 0 & 0.0006 & 242 \\
\hline fixation-regions & nonalcbev > scrambled & superiorfrontal & $\mathrm{R}$ & -3.143 & 8241 & 206.58 & 16 & 53.9 & 24.6 & 0.0003 & 0 & 0.0006 & 313 \\
\hline fixation-regions & nonalcbev > scrambled & caudalmiddlefr & $\mathrm{R}$ & -2.747 & 29029 & 155.88 & 27.8 & 19.3 & 42.7 & 0.0003 & 0 & 0.0006 & 297 \\
\hline task-r & alcbe & superi & $\mathrm{L}$ & -1.996 & 64642 & 333.28 & -22.2 & -84.9 & 23.6 & 0.00927 & 0.00718 & 0.01136 & 479 \\
\hline ask-regions & alcbev > scrambled & fusiform & $\mathrm{R}$ & -4.208 & 114347 & 1011.41 & 31.8 & -74.4 & -11.2 & 0.0003 & 0 & 0.0006 & 1562 \\
\hline sk-regions & alcbev $>$ & (о) & $\mathrm{D}$ & -2.983 & 157893 & 409.83 & 21.3 & -83.2 & 20.7 & 0.0012 & 6 & 0.0021 & 627 \\
\hline task -1 & alcbe & oual & $\mathrm{R}$ & -2.804 & 79452 & 375.68 & 6.9 & -68.3 & 2.4 & 0.0027 & 0.0015 & 0.00389 & 473 \\
\hline ions & alcbe & tal & $\mathrm{R}$ & -2.679 & 93240 & 261.8 & 24.9 & -60.1 & 48 & 264 & 0.02882 & 0.03645 & 553 \\
\hline ask-regions & nonalcbev > scrambled & etal & $\mathrm{L}$ & -2.534 & 120369 & 478.5 & -17 & -88.7 & 20.5 & 0.0003 & 0 & 0.0006 & 709 \\
\hline task-regions & nonalcbev $>$ scrambled & lateralo & $\mathrm{R}$ & -3.465 & 132542 & 658.32 & 29.9 & -85.6 & -12.5 & 0.0003 & 0 & 0.0006 & 985 \\
\hline task-regions & nonalcbev > scrambled & & $\mathrm{R}$ & -261 & 130192 & 242.3 & 199 & -79.4 & 43 & & 511 & 0.02204 & 404 \\
\hline task-regions & nonalcbev > scrambled & al & $\mathrm{R}$ & -2.437 & 61711 & 238.46 & 21.3 & -87.7 & 18 & 0.0 & 0.01789 & 0.0244 & 346 \\
\hline task-regions & scrambled $>$ nonalcbev & superiorfrontal & $\mathrm{L}$ & -3.795 & 4781 & 140.18 & -13.4 & 32.7 & 24.3 & 0.00718 & 0.00539 & 0.00897 & 238 \\
\hline task-regions & scrambled > nonalcbev & rostralmiddlefro & $\mathrm{L}$ & -3.026 & 116230 & 173.7 & -31.1 & 48.1 & 7.1 & & 0.0006 & 0.0021 & 258 \\
\hline task-1 & lcbev & & $\mathrm{L}$ & -1 & 3483 & 148 & -12 & 18.5 & 36.9 & 449 & 0.003 & 0.00599 & 327 \\
\hline task-regions & scrambled $>$ nonalcbev & rostralmiddlefront & $\mathrm{L}$ & -1.861 & 36306 & 136.94 & -36.8 & 31.1 & 29.6 & 0.00897 & 0.00688 & 0.01106 & 200 \\
\hline & & & $\mathrm{L}$ & & & & -8.9 & 30.4 & 32 & & & 4724 & 180 \\
\hline task-regions & nonalcbev > alcbev & superiorfrontal & $\mathrm{L}$ & 2.283 & 110024 & 120.81 & -12.3 & 17.2 & 36.8 & 0.01403 & 0.01136 & 0.01671 & 290 \\
\hline
\end{tabular}

Annotations (from the peak voxel location in the Desikan-Killiany atlas) are shown separately for each of the 22 clusters with significant Group-by-Gender interactions for distractor contrasts. The clusters reported can be understood to span multiple functional regions (Woo et al., 2014). That is, they are not limited to a single region, as reported by the maximal vertex or voxel. Abbreviations: $\mathrm{Hemi}=$ hemisphere; Max $=$ maximum $-\log _{10}(p$-value $)$ for group comparison in the cluster; VtxMax = vertex number at the maximum; Size = surface area of cluster; MNIX, MNIY, and MNIZ = Montreal Neurological Institute 305-subject template coordinates X, Y, and Z for the maximum vertex; CWP = cluster-wise $p$-value further corrected for the three spaces of left cortex, right cortex, and volume; CWPLow and CWPHi $=90 \%$ confidence intervals for CWP; NVtxs $=$ number of vertices in the cluster; alcbev $=$ alcoholic beverages; nonalcbev $=$ nonalcoholic beverages; $\mathrm{L}=$ left hemisphere; $\mathrm{R}=$ right hemisphere. See Table 2 for a summary of the cluster information provided here. 


\begin{tabular}{|c|c|c|c|c|c|c|c|c|c|c|c|c|}
\hline Fixation Masking & Contrast & Annotation & Hemi & $\operatorname{Max}$ & VtxMax & Size $\left(\mathrm{mm}^{\wedge} 2\right)$ & MNIX & MNIY & MNIZ & CWP & CWPLow & CWPHi NVtxs \\
\hline fixation-regions & alcbev > scrambled & inferiorparietal & $\mathrm{L}$ & 4.631 & 58681 & 489.84 & -50.5 & -55 & 23.8 & 0.0003 & 0 & $0.0006 \quad 1036$ \\
\hline fixation-regions & nonalcbev > scrambled & isthmuscingulate & $\mathrm{L}$ & 1.92 & 93859 & 136.3 & -14.5 & -53.6 & 7.6 & 0.00748 & 0.00569 & 0.00927325 \\
\hline task-regions & alcbev > nonalcbev & bankssts & $\mathrm{L}$ & 3.748 & 1233 & 327.69 & -50.5 & -38.3 & 2.9 & 0.0003 & 0 & 0.0006773 \\
\hline task-regions & alcbev > nonalcbev & lateralorbitofrontal & $\mathrm{L}$ & 3.411 & 67740 & 392.95 & -42.1 & 27.4 & -14.3 & 0.0003 & 0 & 0.0006737 \\
\hline task-regions & alcbev > nonalcbev & superiorparietal & $\mathrm{L}$ & 2.862 & 123629 & 420.39 & -30.7 & -50.7 & 49.5 & 0.0003 & 0 & 0.0006950 \\
\hline task-regions & alcbev > nonalcbev & superiortemporal & $\mathrm{R}$ & 2.818 & 12074 & 230.32 & 46.6 & -27.1 & -2.4 & 0.0006 & 0 & 0.0012597 \\
\hline task-regions & alcbev > nonalcbev & inferiorparietal & $\mathrm{R}$ & 2.766 & 31474 & 167.47 & 40 & -74.7 & 11.1 & 0.017 & 0.01403 & 0.01997266 \\
\hline task-regions & alcbev > nonalcbev & parsorbitalis & $\mathrm{R}$ & 2.692 & 27157 & 219.37 & 47.2 & 36.2 & -10.5 & 0.0009 & 0.0003 & $0.0015 \quad 298$ \\
\hline task-regions & alcbev > nonalcbev & lateraloccipital & $\mathrm{R}$ & 2.249 & 56055 & 346.42 & 42.2 & -72 & -5.8 & 0.0003 & 0 & 0.0006461 \\
\hline task-regions & alcbev > scrambled & superiortemporal & $\mathrm{L}$ & 4.221 & 86665 & 439.74 & -47.3 & -31.5 & -3.3 & 0.00449 & 0.003 & 0.005991029 \\
\hline task-regions & alcbev > scrambled & superiorfrontal & $\mathrm{L}$ & 3.663 & 129715 & 522.55 & -7.2 & 24.4 & 46.7 & 0.0015 & 0.0006 & 0.0024919 \\
\hline task-regions & alcbev > scrambled & parsopercularis & $\mathrm{L}$ & 3.155 & 25042 & 876.27 & -45.5 & 17.6 & 20.3 & 0.0003 & 0 & $0.0006 \quad 1611$ \\
\hline task-regions & alcbev > scrambled & parsorbitalis & $\mathrm{L}$ & 2.208 & 10768 & 301.1 & -45.3 & 31.4 & -13.3 & 0.04578 & 0.04112 & 0.05043621 \\
\hline task-regions & alcbev > scrambled & superiorfrontal & $\mathrm{R}$ & 3.989 & 80231 & 427 & 11.6 & 20.7 & 57.1 & 0.0003 & 0 & $0.0006 \quad 844$ \\
\hline task-regions & alcbev > scrambled & caudalmiddlefrontal & $\mathrm{R}$ & 1.867 & 100338 & 259.44 & 38.9 & 7.5 & 55 & 0.03088 & 0.02705 & 0.03469461 \\
\hline
\end{tabular}

\section{Table A5. Cortical cluster characteristics for significant comparisons between ALC and} $920 \quad$ NC groups.

921 The activation levels for all contrasts were significantly greater for the ALC group than for the NC group.

922 Annotations (from the peak voxel location in the Desikan-Killiany atlas) are shown separately for each of the 15

923 clusters with significant group comparisons for distractor contrasts. The clusters reported can be understood to span 924 multiple functional regions (Woo et al., 2014). That is, they are not limited to a single region, as reported by the

925 maximal vertex or voxel. Abbreviations: $\mathrm{Hemi}=$ hemisphere; $\mathrm{Max}=\operatorname{maximum}-\log _{10}(p$-value $)$ for group

926 comparison in the cluster; VtxMax = vertex number at the maximum; Size = surface area of cluster; MNIX, MNIY,

927 and MNIZ = Montreal Neurological Institute 305-subject template coordinates X, Y, and Z for the maximum vertex;

928 CWP $=$ cluster-wise $p$-value further corrected for the three spaces of left cortex, right cortex, and volume; CWPLow 929 and CWPHi $=90 \%$ confidence intervals for CWP; NVtxs = number of vertices in the cluster; alcbev = alcoholic

930 beverages; nonalcbev $=$ nonalcoholic beverages; $\mathrm{L}=$ left hemisphere; $\mathrm{R}=$ right hemisphere; bankssts $=$ banks of the 931 superior temporal sulcus. 


\section{References}

936 Alba-Ferrara, L., Müller-Oehring, E.M., Sullivan, E.V., Pfefferbaum, A., \& Schulte, T. (2016) Brain

937 responses to emotional salience and reward in alcohol use disorder. Brain Imaging Behav., 10, 136-

$938 \quad 146$.

939 Aron, A.R., Robbins, T.W., \& Poldrack, R.A. (2004) Inhibition and the right inferior frontal cortex.

$940 \quad$ Trends Cogn. Sci., 8, 170-177.

941 Avena, N.M., Rada, P., \& Hoebel, B.G. (2008) Evidence for sugar addiction: behavioral and

942 neurochemical effects of intermittent, excessive sugar intake. Neurosci. Biobehav. Rev., 32, $20-39$.

943 Beaty, R.E., Benedek, M., Silvia, P.J., \& Schacter, D.L. (2016) Creative Cognition and Brain Network

944 Dynamics. Trends Cogn. Sci., 20, 87-95.

945 Becker, J.B., McClellan, M.L., \& Reed, B.G. (2017) Sex differences, gender and addiction. J. Neurosci.

$946 \quad$ Res., 95, 136-147.

947 Bednarski, S.R., Zhang, S., Hong, K.-I., Sinha, R., Rounsaville, B.J., \& Li, C.-S.R. (2011) Deficits in

948 default mode network activity preceding error in cocaine dependent individuals. Drug Alcohol

949 Depend., 119, e51-e57.

950 Benishek, L.A., Bieschke, K.J., Stöffelmayr, B.E., Mavis, B.E., \& Humphreys, K.A. (1992) Gender

951 differences in depression and anxiety among alcoholics. J. Subst. Abuse, 4, 235-245.

952 Bordnick, P.S., Traylor, A., Copp, H.L., Graap, K.M., Carter, B., Ferrer, M., \& Walton, A.P. (2008)

953 Assessing reactivity to virtual reality alcohol based cues. Addict. Behav., 33, 743-756.

954 Briggs, G.G. \& Nebes, R.D. (1975) Patterns of hand preference in a student population. Cortex, 11, 230955238.

956 Brighton, R., Moxham, L., \& Traynor, V. (2016) Women and Alcohol Use Disorders: Factors That Lead 957 to Harm. J. Addict. Nurs., 27, 205-213.

958 Broyd, S.J., Demanuele, C., Debener, S., Helps, S.K., James, C.J., \& Sonuga-Barke, E.J.S. (2009) 
Default-mode brain dysfunction in mental disorders: a systematic review. Neurosci. Biobehav. Rev., 33, 279-296.

Buckner, R.L. \& DiNicola, L.M. (2019) The brain's default network: updated anatomy, physiology and evolving insights. Nat. Rev. Neurosci., 20, 593-608.

963 Cahalan, D., Cisin, I.H., \& Crossley, H.M. (1969) American drinking practices: A national study of 964 drinking behavior and attitudes. Monographs of the Rutgers Center of Alcohol Studies, 6, 260.

965 Carter, B.L. \& Tiffany, S.T. (1999) Meta-analysis of cue-reactivity in addiction research. Addiction, 94, $966 \quad 327-340$.

967 Chanraud, S., Pitel, A.-L., Pfefferbaum, A., \& Sullivan, E.V. (2011) Disruption of functional connectivity 968 of the default-mode network in alcoholism. Cereb. Cortex, 21, 2272-2281.

969 Clapp, W.C., Rubens, M.T., \& Gazzaley, A. (2010) Mechanisms of working memory disruption by external interference. Cereb. Cortex, 20, 859-872.

971 Clark, U.S., Oscar-Berman, M., Shagrin, B., \& Pencina, M. (2007) Alcoholism and judgments of 972 affective stimuli. Neuropsychology, 21, 346-362.

973 Dale, A.M. (1999) Optimal experimental design for event-related fMRI. Hum. Brain Mapp., 8, $109-114$.

974 Dale, A.M., Fischl, B., \& Sereno, M.I. (1999) Cortical surface-based analysis. I. Segmentation and 975 surface reconstruction. Neuroimage, 9, 179-194.

976 Desikan, R.S., Ségonne, F., Fischl, B., Quinn, B.T., Dickerson, B.C., Blacker, D., Buckner, R.L., Dale, 977 A.M., Maguire, R.P., Hyman, B.T., Albert, M.S., \& Killiany, R.J. (2006) An automated labeling 978 system for subdividing the human cerebral cortex on MRI scans into gyral based regions of interest. $979 \quad$ Neuroimage, 31, 968-980.

980 Destrieux, C., Fischl, B., Dale, A., \& Halgren, E. (2010) Automatic parcellation of human cortical gyri 981 and sulci using standard anatomical nomenclature. Neuroimage, 53, 1-15.

982 Dolcos, F., LaBar, K.S., \& Cabeza, R. (2005) Remembering one year later: role of the amygdala and the 983 medial temporal lobe memory system in retrieving emotional memories. Proc. Natl. Acad. Sci. U. S. 984 A., 102, 2626-2631. 
Dolcos, F. \& McCarthy, G. (2006) Brain systems mediating cognitive interference by emotional distraction. J. Neurosci., 26, 2072-2079.

Eklund, A., Nichols, T.E., \& Knutsson, H. (2016) Cluster failure: Why fMRI inferences for spatial extent have inflated false-positive rates. Proc. Natl. Acad. Sci. U. S. A., 113, 7900-7905.

Fama, R., Le Berre, A.-P., \& Sullivan, E.V. (2020) Alcohol's Unique Effects on Cognition in Women: A 2020 (Re)view to Envision Future Research and Treatment. Alcohol Res., 40, 03.

991 Feldstein Ewing, S.W., Filbey, F.M., Chandler, L.D., \& Hutchison, K.E. (2010) Exploring the relationship between depressive and anxiety symptoms and neuronal response to alcohol cues.

Field, M. \& Cox, W. (2008) Attentional bias in addictive behaviors: A review of its development, causes, and consequences. Drug and Alcohol Dependence, 97, 1-20.

Field, M. \& Eastwood, B. (2005) Experimental manipulation of attentional bias increases the motivation to drink alcohol. Psychopharmacology, 183, 350-357.

Field, M., Marhe, R., \& Franken, I.H.A. (2014) The clinical relevance of attentional bias in substance use disorders. CNS Spectr., 19, 225-230.

Field, M., Mogg, K., Zetteler, J., \& Bradley, B.P. (2004) Attentional biases for alcohol cues in heavy and light social drinkers: the roles of initial orienting and maintained attention. Psychopharmacology, 176, 88-93.

Field, M., Munafò, M.R., \& Franken, I.H.A. (2009) A meta-analytic investigation of the relationship between attentional bias and subjective craving in substance abuse. Psychol. Bull., 135, 589-607.

Fischl, B., Salat, D.H., Busa, E., Albert, M., Dieterich, M., Haselgrove, C., van der Kouwe, A., Killiany, R., Kennedy, D., Klaveness, S., Montillo, A., Makris, N., Rosen, B., \& Dale, A.M. (2002) Whole brain segmentation: automated labeling of neuroanatomical structures in the human brain. Neuron, 33, 341-355.

1010

Fischl, B., Sereno, M.I., Tootell, R.B.H., \& Dale, A.M. (1999) High-resolution intersubject averaging and a coordinate system for the cortical surface. Human Brain Mapping, 8, 272-284. 
1011 Flannery, B.A., Volpicelli, J.R., \& Pettinati, H.M. (1999) Psychometric properties of the Penn Alcohol 1012 Craving Scale. Alcohol. Clin. Exp. Res., 23, 1289-1295.

1013 Franken, I.H.A. (2003) Drug craving and addiction: integrating psychological and neuropsychopharmacological approaches. Prog. Neuropsychopharmacol. Biol. Psychiatry, 27, 563579.

Fryer, S.L., Jorgensen, K.W., Yetter, E.J., Daurignac, E.C., Watson, T.D., Shanbhag, H., Krystal, J.H., \&

1019 Garber, A.K. \& Lustig, R.H. (2011) Is fast food addictive? Curr. Drug Abuse Rev., 4, 146-162.

1020 George, M.S., Anton, R.F., Bloomer, C., Teneback, C., Drobes, D.J., Lorberbaum, J.P., Nahas, Z., \& Vincent, D.J. (2001) Activation of prefrontal cortex and anterior thalamus in alcoholic subjects on exposure to alcohol-specific cues. Arch. Gen. Psychiatry, 58, 345-352.

Goldstein, R.Z. \& Volkow, N.D. (2002) Drug addiction and its underlying neurobiological basis: neuroimaging evidence for the involvement of the frontal cortex. Am. J. Psychiatry, 159, 1642-1652.

Goldstein, R.Z. \& Volkow, N.D. (2011) Dysfunction of the prefrontal cortex in addiction: neuroimaging findings and clinical implications. Nature Reviews Neuroscience, 12, 652-669.

Greve, D.N. \& Fischl, B. (2009) Accurate and robust brain image alignment using boundary-based registration. Neuroimage, $\mathbf{4 8 , 6 3 - 7 2 . ~}$

1031 Hamilton, M. (1960) A rating scale for depression. J. Neurol. Neurosurg. Psychiatry, 23, 56-62.

1032 Heilbronner, S.R. \& Hayden, B.Y. (2016) Dorsal anterior cingulate cortex: A bottom-up view. Annu. Rev. $1033 \quad$ Neurosci., 39, 149-170.

1034 Heinz, A., Wrase, J., Kahnt, T., Beck, A., Bromand, Z., Grüsser, S.M., Kienast, T., Smolka, M.N., Flor, 1035 H., \& Mann, K. (2007) Brain activation elicited by affectively positive stimuli is associated with a lower risk of relapse in detoxified alcoholic subjects. Alcohol. Clin. Exp. Res., 31, 1138-1147. 
Hoffman, L.A., Lewis, B., \& Nixon, S.J. (2019) Neurophysiological and interpersonal correlates of emotional face processing in Alcohol Use Disorder. Alcohol. Clin. Exp. Res., 43.

Holdnack, J.A. \& Drozdick, L.W. (2010) CHAPTER 9 - Using WAIS-IV with WMS-IV. In Weiss, L.G., Saklofske, D.H., Coalson, D.L., \& Raiford, S.E. (eds), WAIS-IV Clinical Use and Interpretation. Academic Press, San Diego, pp. 237-283.

Koob, G.F. \& Volkow, N.D. (2010) Neurocircuitry of addiction. Neuropsychopharmacology, 35, 217238.

Koshino, H., Minamoto, T., Ikeda, T., Osaka, M., Otsuka, Y., \& Osaka, N. (2011) Anterior medial prefrontal cortex exhibits activation during task preparation but deactivation during task execution. PLoS One, 6, e22909.

Kunz, S., Beblo, T., Driessen, M., \& Woermann, F. (2008) fMRI of alcohol craving after individual cues: a follow-up case report. Neurocase, 14, 343-346.

LeDoux, J.E. (1996) The Emotional Brain: The Mysterious Underpinnings of Emotional Life. Simon \& Schuster.

Lewis, B., Price, J.L., Garcia, C.C., \& Nixon, S.J. (2019) Emotional Face Processing among TreatmentSeeking Individuals with Alcohol Use Disorders: Investigating Sex Differences and Relationships with Interpersonal Functioning. Alcohol Alcohol, 54, 361-369.

Loeber, S., Vollstädt-Klein, S., von der Goltz, C., Flor, H., Mann, K., \& Kiefer, F. (2009) Attentional bias in alcohol-dependent patients: the role of chronicity and executive functioning. Addict. Biol., 14,

\section{2}

$$
\text { 194-203. }
$$

Lubman, D.I. (2007) Addiction neuroscience and its relevance to clinical practice. Drug Alcohol Rev., 26,

$$
1-2 .
$$


Luhar, R.B., Sawyer, K.S., Gravitz, Z., Ruiz, S.M., \& Oscar-Berman, M. (2013) Brain volumes and neuropsychological performance are related to current smoking and alcoholism history. Neuropsychiatr. Dis. Treat., 9, 1767-1784.

Makris, N., Oscar-Berman, M., Jaffin, S.K., Hodge, S.M., Kennedy, D.N., Caviness, V.S., Marinkovic, K., Breiter, H.C., Gasic, G.P., \& Harris, G.J. (2008) Decreased volume of the brain reward system in alcoholism. Biological Psychiatry, 64, 192-202.

1070 gender differences in alcohol dependence: Are women more vulnerable? Alcoholism: Clinical \&

Margulies, D.S., Ghosh, S.S., Goulas, A., Falkiewicz, M., Huntenburg, J.M., Langs, G., Bezgin, G., Eickhoff, S.B., Castellanos, F.X., Petrides, M., Jefferies, E., \& Smallwood, J. (2016) Situating the default-mode network along a principal gradient of macroscale cortical organization. Proc. Natl. Acad. Sci. U. S. A., 113, 12574-12579. Res., 33, 1880-1892.

Menon, V. (2011) Large-scale brain networks and psychopathology: a unifying triple network model.

1081 Merrill, J.E. \& Read, J.P. (2010) Motivational pathways to unique types of alcohol consequences. Psychol. Addict. Behav., 24, 705-711.

1083 Mosher Ruiz, S., Oscar-Berman, M., Kemppainen, M.I., Valmas, M.M., \& Sawyer, K.S. (2017)

1084 Associations between personality and drinking motives among abstinent adult alcoholic men and 1085 women. Alcohol Alcohol, 52, 496-505.

1086 Myrick, H., Anton, R.F., Li, X., Henderson, S., Drobes, D., Voronin, K., \& George, M.S. (2004)

1087 Differential brain activity in alcoholics and social drinkers to alcohol cues: relationship to craving. 1088 Neuropsychopharmacology, 29, 393-402. 
Myrick, H., Anton, R.F., Li, X., Henderson, S., Randall, P.K., \& Voronin, K. (2008) Effect of naltrexone and ondansetron on alcohol cue-induced activation of the ventral striatum in alcohol-dependent people. Arch. Gen. Psychiatry, 65, 466-475.

1092 Oscar-Berman, M., Hancock, M., Mildworf, B., Hutner, N., \& Weber, D.A. (1990) Emotional perception and memory in alcoholism and aging. Alcohol. Clin. Exp. Res., 14, 383-393.

1094 Oscar-Berman, M. \& Maleki, N. (2019) Alcohol Dementia, Wernicke's Encephalopathy, and Korsakoff's 1095 Syndrome. In Michael L. Alosco and Robert A. Stern (ed), The Oxford Handbook of Adult Cognitive 1096 Disorders. Oxford University Press, pp. 743-758.

1097 Oscar-Berman, M., Ruiz, S.M., Marinkovic, K., Valmas, M.M., Harris, G.J., \& Sawyer, K.S. (2019)

1098 Brain responsivity to emotional faces differs in alcoholic men and women. bioRxiv, 496166.

1099 Oscar-Berman, M., Valmas, M.M., Sawyer, K.S., Ruiz, S.M., Luhar, R.B., \& Gravitz, Z.R. (2014)

1100 Profiles of impaired, spared, and recovered neuropsychologic processes in alcoholism. Handb. Clin. $1101 \quad$ Neurol., 125, 183-210.

1102 Philippot, P., Kornreich, C., Blairy, S., Baert, I., Den Dulk, A., Le Bon, O., Streel, E., Hess, U., Pelc, I., \& 1103 Verbanck, P. (1999) Alcoholics' deficits in the decoding of emotional facial expression. Alcohol. 1104 Clin. Exp. Res., 23, 1031-1038.

1105 Pourtois, G., Schwartz, S., Spiridon, M., Martuzzi, R., \& Vuilleumier, P. (2009) Object representations 1106 for multiple visual categories overlap in lateral occipital and medial fusiform cortex. Cereb. Cortex, $1107 \quad$ 19, 1806-1819.

1108 Ray, S., Hanson, C., Hanson, S.J., \& Bates, M.E. (2010) fMRI BOLD response in high-risk college 1109 students (Part 1): during exposure to alcohol, marijuana, polydrug and emotional picture cues. $1110 \quad$ Alcohol Alcohol, 45, 437-443.

1111 Reinhard, I., Leménager, T., Fauth-Bühler, M., Hermann, D., Hoffmann, S., Heinz, A., Kiefer, F., 1112 Smolka, M.N., Wellek, S., Mann, K., \& Vollstädt-Klein, S. (2015) A comparison of region-of1113 interest measures for extracting whole brain data using survival analysis in alcoholism as an 1114 example. J. Neurosci. Methods, 242, 58-64. 
1115 Rivas-Grajales, A.M., Sawyer, K.S., Karmacharya, S., Papadimitriou, G., Camprodon, J.A., Harris, G.J.,

1116 Kubicki, M., Oscar-Berman, M., \& Makris, N. (2018) Sexually dimorphic structural abnormalities in

1117 major connections of the medial forebrain bundle in alcoholism. NeuroImage: Clinical, 19, 98-105.

1118 Robins, L.N., Cottler, L.B., Bucholz, K.K., Compton, W.M., North, C.S., \& Rourke, K. (2000)

1119 Computerized Diagnostic Interview Schedule for the DSM-IV (C DIS-IV).

1120 Rubio, G., Martínez-Gras, I., Ponce, G., Quinto, R., Jurado, R., \& Jiménez-Arriero, M.Á. (2013)

1121 [Integration of self-guidance groups for relatives in a public program of alcoholism treatment].

1122 Adicciones, 25, 37-44.

1123 Ruiz, S.M. \& Oscar-Berman, M. (2015) Gender and alcohol abuse: history and sociology. In Martin, S.C.

1124 (ed), Gender and Alcohol Abuse: History and Sociology, The SAGE Encyclopedia of Alcohol:

1125 Social, Cultural, and Historical Perspectives. Sage Publications Los Angeles, pp. 586-591.

1126 Ruiz, S.M., Oscar-Berman, M., Sawyer, K.S., Valmas, M.M., Urban, T., \& Harris, G.J. (2013) Drinking

1127 history associations with regional white matter volumes in alcoholic men and women. Alcohol. Clin.

1128 Exp. Res., 37, 110-122.

1129 Ryan, F. (2002) Attentional bias and alcohol dependence: a controlled study using the modified stroop 1130 paradigm. Addict. Behav., 27, 471-482.

1131 Saraceno, L., Heron, J., Munafò, M., Craddock, N., \& van den Bree, M.B.M. (2012) The relationship between childhood depressive symptoms and problem alcohol use in early adolescence: findings

1134 Sawyer, K.S., Adra, N., Salz, D.M., Kemppainen, M.I., Ruiz, S.M., Harris, G.J., \& Oscar-Berman, M. 1135 (2020) Hippocampal subfield volumes in abstinent men and women with a history of alcohol use 1136 disorder. PLoS One, 15, e0236641.

1137 Sawyer, K.S., Maleki, N., Papadimitriou, G., Makris, N., Oscar-Berman, M., \& Harris, G.J. (2018)

1138 Cerebral white matter sex dimorphism in alcoholism: a diffusion tensor imaging study.

$1139 \quad$ Neuropsychopharmacology, 43, 1876-1883.

1140 Sawyer, K.S., Maleki, N., Urban, T., Marinkovic, K., Karson, S., Ruiz, S.M., Harris, G.J., \& Oscar- 
Berman, M. (2019) Alcoholism gender differences in brain responsivity to emotional stimuli. Elife,

1143 Sawyer, K.S., Oscar-Berman, M., Barthelemy, O.J., Papadimitriou, G.M., Harris, G.J., \& Makris, N.

1144 (2017) Gender dimorphism of brain reward system volumes in alcoholism. Psychiatry Res

$1145 \quad$ Neuroimaging, 263, 15-25.

1146 Sawyer, K.S., Poey, A., Ruiz, S.M., Marinkovic, K., \& Oscar-Berman, M. (2015) Measures of skin

1147 conductance and heart rate in alcoholic men and women during memory performance. PeerJ, $\mathbf{3}$,

1148 e941.

1149 Schacht, J.P., Anton, R.F., \& Myrick, H. (2013) Functional neuroimaging studies of alcohol cue

1150 reactivity: a quantitative meta-analysis and systematic review: Alcohol cue imaging. Addict. Biol.,

$1151 \quad \mathbf{1 8}, 121-133$.

1152 Schneider, F., Habel, U., Wagner, M., Franke, P., Salloum, J.B., Shah, N.J., Toni, I., Sulzbach, C., Hönig,

1153 K., Maier, W., Gaebel, W., \& Zilles, K. (2001) Subcortical correlates of craving in recently abstinent

1154 alcoholic patients. Am. J. Psychiatry, 158, 1075-1083.

1155 Schulte, M.T., Ramo, D., \& Brown, S.A. (2009) Gender differences in factors influencing alcohol use and 1156 drinking progression among adolescents. Clin. Psychol. Rev., 29, 535-547.

1157 Ségonne, F., Dale, A.M., Busa, E., Glessner, M., Salat, D., Hahn, H.K., \& Fischl, B. (2004) A hybrid 1158 approach to the skull stripping problem in MRI. NeuroImage, 22, 1060-1075.

1159 Seitz, J., Sawyer, K.S., Papadimitriou, G., Oscar-Berman, M., Ng, I., Kubicki, A., Mouradian, P., Ruiz, 1160 S.M., Kubicki, M., Harris, G.J., \& Makris, N. (2017) Alcoholism and sexual dimorphism in the 1161 middle longitudinal fascicle: a pilot study. Brain Imaging Behav., 11, 1006-1017.

1162 Seo, D., Jia, Z., Lacadie, C.M., Tsou, K.A., Bergquist, K., \& Sinha, R. (2011) Sex differences in neural 1163 responses to stress and alcohol context cues. Human Brain Mapping, 32, 1998-2013.

1164 Sharma, D., Albery, I.P., \& Cook, C. (2001) Selective attentional bias to alcohol related stimuli in 1165 problem drinkers and non-problem drinkers. Addiction, 96, 285-295.

1166 Shields, C.N. \& Gremel, C.M. (2020) Review of orbitofrontal cortex in alcohol dependence: A disrupted 
cognitive map? Alcohol. Clin. Exp. Res., 44, 1952-1964.

1168 Sinha, R., Fox, H.C., Hong, K.A., Bergquist, K., Bhagwagar, Z., \& Siedlarz, K.M. (2009) Enhanced negative emotion and alcohol craving, and altered physiological responses following stress and cue exposure in alcohol dependent individuals. Neuropsychopharmacology, 34, 1198-1208.

Sled, J.G., Zijdenbos, A.P., \& Evans, A.C. (1998) A nonparametric method for automatic correction of intensity nonuniformity in MRI data. IEEE Transactions on Medical Imaging, 17, 87-97.

Sormaz, M., Murphy, C., Wang, H.-T., Hymers, M., Karapanagiotidis, T., Poerio, G., Margulies, D.S.,

Stritzke, W.G.K., Breiner, M.J., Curtin, J.J., \& Lang, A.R. (2004) Assessment of substance cue reactivity: advances in reliability, specificity, and validity. Psychol. Addict. Behav., 18, 148-159.

Talairach, J. \& Tournoux, P. (1988) Co-Planar Stereotaxic Atlas of the Human Brain: 3-Dimensional Proportional System : An Approach to Cerebral Imaging. G. Thieme, Stuttgart. Brown, S.A. (2003) Neural response to alcohol stimuli in adolescents with alcohol use disorder. Arch. Gen. Psychiatry, 60, 727-735.

Thesen, S., Heid, O., Mueller, E., \& Schad, L.R. (2000) Prospective acquisition correction for head motion with image-based tracking for real-time fMRI. Magn. Reson. Med., 44, 457-465.

Thompson-Schill, S.L., Jonides, J., Marshuetz, C., Smith, E.E., D’Esposito, M., Kan, I.P., Knight, R.T., \& Swick, D. (2002) Effects of frontal lobe damage on interference effects in working memory. Cogn. Affect. Behav. Neurosci., 2, 109-120.

Tops, M., Boksem, M.A.S., Quirin, M., IJzerman, H., \& Koole, S.L. (2014) Internally directed cognition and mindfulness: an integrative perspective derived from predictive and reactive control systems theory. Front. Psychol., 5, 429.

Townshend, J.M. \& Duka, T. (2001) Attentional bias associated with alcohol cues: differences between heavy and occasional social drinkers. Psychopharmacology, 157, 67-74. 
1193 Uddin, L.Q., Yeo, B.T.T., \& Spreng, R.N. (2019) Towards a universal taxonomy of macro-scale

1194 functional human brain networks. Brain Topogr., 32, 926-942.

1195 Verplaetse, T.L., Cosgrove, K.P., Tanabe, J., \& McKee, S.A. (2021) Sex/gender differences in brain

1196 function and structure in alcohol use: A narrative review of neuroimaging findings over the last 10

1197 years. J. Neurosci. Res., 99, 309-323.

1198 Volkow, N.D., Wang, G.-J., Tomasi, D., \& Baler, R.D. (2013) The addictive dimensionality of obesity.

1199 Biological Psychiatry, 73, 811-818.

1200 Vollstädt-Klein, S., Loeber, S., Kirsch, M., Bach, P., Richter, A., Bühler, M., von der Goltz, C., Hermann,

1201 D., Mann, K., \& Kiefer, F. (2011) Effects of cue-exposure treatment on neural cue reactivity in

1202 alcohol dependence: a randomized trial. Biol. Psychiatry, 69, 1060-1066.

1203 Wechsler, D. (1997) WAIS-III, Wechsler Adult Intelligence Scale, Third Edition: WMS-III, Wechsler

1204 Memory Scale, Third Edition: Technical Manual. Psychological Corporation, San Antonio, TX.

1205 Wiers, C.E., Stelzel, C., Park, S.Q., Gawron, C.K., Ludwig, V.U., Gutwinski, S., Heinz, A., Lindenmeyer,

1206 J., Wiers, R.W., Walter, H., \& Bermpohl, F. (2014) Neural correlates of alcohol-approach bias in

1207 alcohol addiction: the spirit is willing but the flesh is weak for spirits. Neuropsychopharmacology,

$1208 \quad 39,688-697$.

1209 Woo, C.-W., Krishnan, A., \& Wager, T.D. (2014) Cluster-extent based thresholding in fMRI analyses:

1210 pitfalls and recommendations. Neuroimage, 91, 412-419.

1211 World Health Organization (2019) Global Status Report on Alcohol and Health 2018. World Health

1212 Organization.

1213 Wrase, J., Grüsser, S.M., Klein, S., Diener, C., Hermann, D., Flor, H., Mann, K., Braus, D.F., \& Heinz, 1214 A. (2002) Development of alcohol-associated cues and cue-induced brain activation in alcoholics. $1215 \quad$ Eur. Psychiatry, 17, 287-291.

1216 Yeo, B.T.T., Krienen, F.M., Eickhoff, S.B., Yaakub, S.N., Fox, P.T., Buckner, R.L., Asplund, C.L., \&

1217 Chee, M.W.L. (2016) Functional specialization and flexibility in human association cortex. Cereb.

$1218 \quad$ Cortex, 26, 465. 
1219 Zhang, R. \& Volkow, N.D. (2019) Brain default-mode network dysfunction in addiction. Neuroimage,

$1220 \quad 200,313-331$.

1221 Zhou, H.-X., Chen, X., Shen, Y.-Q., Li, L., Chen, N.-X., Zhu, Z.-C., Castellanos, F.X., \& Yan, C.-G.

1222 (2020) Rumination and the default mode network: Meta-analysis of brain imaging studies and

1223 implications for depression. Neuroimage, 206, 116287. 